\title{
Influence of cut-off truncation and artificial periodicity of electrostatic interactions in molecular simulations of solvated ions: A continuum electrostatics study
}

\author{
Michael Bergdorf, Christine Peter, and Philippe H. Hünenberger ${ }^{a)}$ \\ Laboratorium für Physikalische Chemie, ETH Zürich, CH-8093 Zürich, Switzerland
}

(Received 13 May 2003; accepted 6 August 2003)

\begin{abstract}
A new algorithm relying on finite integration is presented that solves the equations of continuum electrostatics for truncated (and possibly reaction-field corrected) solute-solvent and solventsolvent interactions under either nonperiodic or periodic boundary conditions. After testing and validation by comparison with existing methods, the algorithm is applied to investigate the effect of cut-off truncation and artificial periodicity in explicit-solvent simulations of ionic solvation and ion-ion interactions. Both cut-off truncation and artificial periodicity significantly alter the polarization around a spherical ion and thus, its solvation free energy. The nature and magnitude of the two perturbations are analyzed in details, and correction terms are proposed for both effects. Cut-off truncation is also shown to induce strong alterations in the potential of mean force for ion-ion interaction. These observations help to rationalize artifacts previously observed in explicitsolvent simulations, namely spurious features in the radial distribution functions close to the cut-off distance and alterations in the relative stabilities of contact, solvent-separated and free ion pairs. (C) 2003 American Institute of Physics.
\end{abstract}

[DOI: $10.1063 / 1.1614202]$

\section{INTRODUCTION}

Computer simulation with an explicit representation of the solvent molecules has become a standard tool for investigating the structure, dynamics, and function of (bio-)molecules in solution. ${ }^{1-6}$ However, due to important computational costs, the system sizes that are accessible to such simulations remain truly microscopic (typically $<1000 \mathrm{~nm}^{3}$ ). As a direct consequence, the longest-range $(>5 \mathrm{~nm})$ component of intermolecular interactions, which is generally dominated by electrostatics, cannot be computed in an exact manner. Unfortunately, because electrostatic interactions are of large magnitude, many simulated observables turn out to be highly sensitive to the treatment of these interactions and, due to their long range, to the boundary conditions used in the simulation (system size and shape, finite versus periodic system). For this reason, the approximate representation of long-range electrostatic interactions in explicit-solvent (bio-)molecular simulations is probably nowadays one of the principal bottlenecks in the accuracy of these methods. Uncontrolled approximations can give rise to important artifacts (so-called finite-size effects), which may strongly impair the reliability of many current simulations. There is thus considerable effort in the scientific community towards the goal of improving the representation of electrostatics in computer simulations.

The vast majority of explicit-solvent (bio-)molecular simulations are carried out under periodic boundary conditions $^{6,7}$ (PBC). In this case, the solute (bio-)molecule is

a) Author to whom correspondence should be addressed. Telephone: +411 632 5503; Fax: +4116321039.

Electronic mail: phil@igc.phys.chem.ethz.ch placed into a computational box (space-filling shape, e.g., rectangular), and the empty volume is filled by solvent molecules. The system considered in the simulation consists of the central box surrounded by an infinite array of periodic copies of itself, which has the advantage of removing any surface distortion associated with a solvent-vacuum boundary. There are essentially three methods to handle electrostatic interactions in simulations under PBC: (i) Straight truncation of the Coulomb interactions at a convenient cutoff distance; ${ }^{6,7}$ (ii) smooth truncation of the Coulomb interactions, e.g., by means of a switching or shifting function ${ }^{8-13}$ or by including a reaction-field correction; ${ }^{14-19}$ (iii) use of lattice-sum methods (Ewald, ${ }^{20} \mathrm{P}^{3} \mathrm{M},{ }^{21}$ or $\mathrm{PME}^{22,23}$ methods). Cut-off truncation reduces the computational costs and the effect of artificial periodicity in simulations. However, straight truncation (ST) represents a very severe approximation, leading to heating as well as important artifacts in simulated properties of liquids, ${ }^{16,24,25}$ solvated ions, ${ }^{26-35}$ ion pairs, ${ }^{9,36-44}$ and biomolecules. ${ }^{45-48}$ Smooth-truncation methods may be applied to reduce the heating caused by the application of a cut-off, but nevertheless retain (and sometimes amplify) a number of the undesirable effects of abrupt truncation. $9,10,27,28,41,48-51$ Furthermore, these methods are generally $a d$ hoc and lack any physical basis. An exception is the inclusion of a Barker-Watts reaction-field correction ${ }^{14-16}$ (RF) to the cut-off truncation. This correction scheme approximately accounts for the mean effect of the medium beyond the cut-off sphere of each particle by assuming that this medium behaves as a homogeneous dielectric continuum of permittivity equal to that of the solvent. Due to the form of the added reaction-field term, the correction effectively acts as a (physically based) switching function in the limit of 
high solvent permittivities. Finally, lattice-sum (LS) methods ${ }^{20-23,52-54}$ rely on Fourier series to describe the longrange component of electrostatic interactions, i.e., they assume that these interactions are exactly periodic within the infinite system. Although LS and RF methods rely on more or less reasonable approximations for dealing with the longrange component of electrostatic interactions, an approximate treatment is certainly preferable to the plain omission of this long-range component, as done in the ST scheme. Nevertheless, some dependence of simulated observables on the cut-off distance or system size has also been evidenced for the LS, ${ }^{19,55-59}$ and $\mathrm{RF}^{27,28,60}$ methods. It is therefore of importance to carefully investigate and compare the properties of the three most common electrostatic schemes (ST, RF, and LS).

A general strategy to analyze finite-size effects and improve electrostatic schemes for explicit-solvent simulations relies on the use of continuum electrostatics. ${ }^{26-28,51,52,56-64}$ In the continuum-electrostatics approach, the solute is treated as a low-dielectric cavity encompassing the solute atomic point charges, and embedded in a dielectric continuum of permittivity equal to that of the solvent. In the classical implementation of the method, ${ }^{65-68}$ the electrostatic potential in the system is computed by numerically solving the Poisson (or Poisson-Boltzmann, in the presence of implicit counterions) equation, giving access to the electrostatic solvation free energy of the solute. Although there is no choice of boundary conditions that adequately mimics an infinitely dilute solution in explicit-solvent simulations, this is not the case in continuum-electrostatics calculations. There, the boundary conditions to solve the Poisson equation are specified in the form of the potential at the surface of the computational box. For a reasonably large solute-wall distance, this potential is well approximated by the solvent-screened Coulomb potential of the solute charges. ${ }^{57}$ In this way, continuum electrostatics can be used to estimate, for a given solute configuration, the electrostatic solvation free energy corresponding to exact (nontruncated) Coulomb interactions (CB) under nonperiodic boundary conditions (NPBC), a good model for the ideal situation of a solute at infinite dilution. This suggests that artifacts linked with the use of approximate electrostatic interactions and periodic boundary conditions in explicit-solvent simulations could be investigated using continuum electrostatics, provided that the method is generalized to these modified interactions and boundary conditions. Such generalizations have recently been developed ${ }^{26-28,51,52,57-64}$ for nearly all types of relevant electrostatic interaction schemes (CB/LS, ST, or RF) and boundary conditions (NPBC or PBC), as summarized in Table I. By comparing, for a given solute configuration, the outcome of a continuum-electrostatics calculation based on modified interactions and boundary conditions with that of another calculation based on CB interactions under NPBC, it is possible to estimate the perturbation $\Delta \Delta G_{\text {solv }}$ of the solvation free energy. The corresponding perturbation $\Delta E_{\text {direct }}$ in the direct electrostatic interaction energy between solute atomic charges is straightforward to calculate. The sum $\Delta \Delta G_{\mathrm{el}}$ of the two contributions represents the perturbation of the electrostatic free energy of the system due to the use of
TABLE I. Generalizations of the continuum-electrostatics approach to modified electrostatic interactions and boundary conditions. The methods have been classified using the codes 3D (problem solved in three dimensions), 1D (problem reduced to a one-dimensional equation by symmetry), analytical (analytical solution available), Poisson (based on solving the Poisson equation), FFT (based on the use of fast Fourier transforms), or direct (based on solving field equations analogous to Eqs. (1) and (6) in realspace).

\begin{tabular}{lll}
\hline \hline Electrostatics & \multicolumn{1}{c}{ NPBC } & \multicolumn{1}{c}{ PBC } \\
\hline CB/LS $^{\mathrm{a}}$ & 3D-Poisson (Ref. 95) & \multicolumn{1}{c}{ 3D-Poisson (Ref. 57) } \\
& 3D-FFT (Refs. 51, 63, and 64) \\
(spherical ion $\left.^{\mathrm{c}}\right)$ & 1D-Analytical (Ref. 86) & 1D-Analytical (Ref. 57) \\
SC,RF $^{\mathrm{d}}$ & 3D-Direct & 3D-Direct \\
& & 3D-FFT (Refs. 51 and 60) \\
(spherical ion $\left.^{\mathrm{c}}\right)$ & 1D-Direct (Refs. 26-28) & - \\
\hline \hline
\end{tabular}

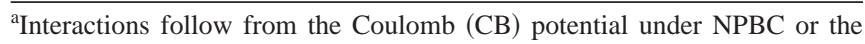
Ewald lattice-sum (LS) potential under PBC.

${ }^{\mathrm{b}}$ First calculation on a biomolecule using a finite-difference algorithm (many alternatives have been proposed, including, e.g., finite-element, boundaryelement and inducible-multipole algorithms).

'Solutions developed for the special case of a single spherical ion.

${ }^{\mathrm{d}}$ Interactions follow from the Coulomb potential truncated at a given cut-off distance, without (ST) or with (RF) the inclusion of a reaction-field correction.

${ }^{\mathrm{e}}$ Developed in the present article.

approximate electrostatics and boundary conditions in the simulation. This procedure is illustrated schematically in Fig. 1 for the specific case of an explicit-solvent simulation employing ST or RF electrostatics under PBC. The quantity $\Delta \Delta G_{\text {el }}$ (possibly evaluated for multiple solute configurations) gives the required information to investigate the nature

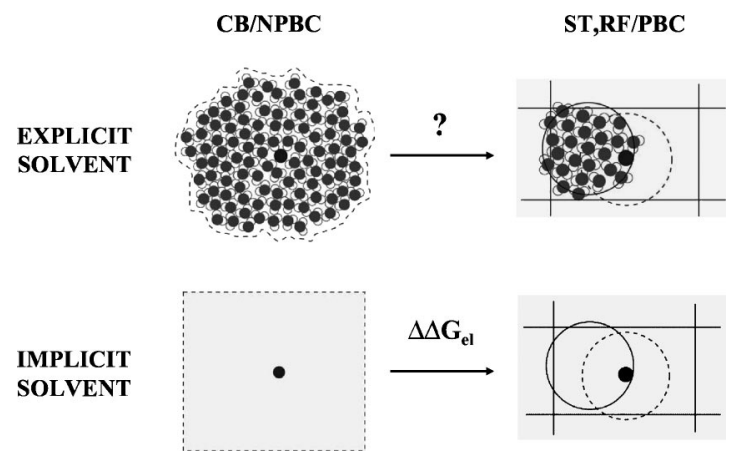

FIG. 1. Schematic illustration of the procedure used to assess, based on continuum electrostatics, artifacts linked with approximate electrostatics and boundary conditions in explicit-solvent simulations. Ideally, an explicitsolvent simulation aiming to describe a solute molecule (symbolized by a black sphere) at infinite dilution should be based on a quasi-macroscopic system under NPBC together with exact CB interactions (top left drawing). Due to computational limitations this is not feasible in practice, and one may simulate instead a system under PBC with ST (or RF) electrostatic interactions (top right drawing). The corresponding perturbation can be evaluated by considering the implicit-solvent analogs of the two cases. Using continuum electrostatics (for a given solute configuration), the solvation free energies and the direct interactions between solute charges can be computed both under CB/NPBC (bottom left drawing; based on a good approximation for the electrostatic potential at the surface of the computational volume) and under ST/PBC or RF/PBC (bottom right drawing). The free-energy difference $\Delta \Delta G_{\mathrm{el}}$ represents the perturbation of the electrostatic free energy induced by the use approximate electrostatics and boundary conditions, and is a key quantity for the analysis of finite-size effects in the explicit-solvent simulation. 
and magnitude of the corresponding artifacts in explicitsolvent simulations.

Since they ignore the discrete nature of the solvent, continuum-electrostatics models have some limitations, including an important sensitivity to empirical model parameters (atomic charges, atomic radii, solute permittivity, exact definition of the solute-solvent boundary), the neglect of nonlinear effects (electrostriction, dielectric saturation), and the neglect of the detailed solvent structure around the solute (structure of the first solvation shells, specific hydrogen bonds). Furthermore, the electrostatic contribution to the solvation free energy should be complemented by a nonpolar contribution, typically assumed to be proportional to the solvent-exposed surface area. However, since the present method relies on the comparison of two closely related continuum-electrostatics calculations involving the same parameters and solute configuration (Fig. 1, bottom drawings), it is likely that errors in the short-range description of solvation cancel out to a large extent. The difference will depend almost exclusively on long-range effects, for which continuum electrostatics can be expected to be accurate. Thus, electrostatic free energy differences from continuum electrostatics should be almost quantitatively transposable to interpret finite-size artifacts in explicit-solvent simulations.

Inspection of Table I reveals one missing entry. There is currently no general continuum-electrostatics method to deal with truncated electrostatic interactions (ST or RF) under NPBC, although a method exists in the special case of a single spherical ion ${ }^{26-28}$ [one-dimensional (1D)-Direct method]. The goal of the present article is to describe and apply a general method based on field equations and a finiteintegration algorithm [three-dimensional (3D)-Direct method]. In addition to dealing with the NPBC case, this new method is also applicable to systems under PBC. However, it scales rather unfavorably with the system size (as $N_{g}^{6}$, where $N_{g}$ is the number of grid points along each Cartesian direction), and can only be used for small systems. Therefore, its application is restricted here to the investigation of the consequences of cut-off truncation and artificial periodicity of electrostatic interactions in molecular simulations of ionic solvation and ion-ion interaction. These systems are very important benchmark systems for evaluating the accuracy of electrostatic interactions in molecular simulations because (i) they offer the simplest context to investigate electrostatic finite-size effects, and (ii) despite the apparent simplicity of the problem, the accurate determination of ionic solvation free energies, $9,19,26-35,55,57,69-79$ and ion-ion potentials of mean force $e^{9,36-44,57,80-84}$ has turned out to be a surprisingly difficult problem.

In the present article, the algorithm is described in details and the influence of various parameters controlling its behavior is investigated. The accuracy of the algorithm is further tested by comparing solvation free energies computed for a single spherical ion to values estimated through the 1D-Direct ${ }^{26-28}$ (NPBC) or 3D-FFT ${ }^{51,60}$ (PBC) methods. Finally, the present 3D-Direct method is applied to investigate the effect of cut-off truncation and artificial periodicity in computer simulations of ionic solvation and ion-ion interactions.

\section{THEORY}

\section{A. Continuum electrostatics}

In the continuum-electrostatics approach, a solutesolvent system is modeled (for a given solute configuration) as a set of solute atomic partial charges embedded in a polarizable medium of heterogeneous dielectric permittivity. Application of the laws of electrostatics within such a system leads to the following expression ${ }^{24,25,51,64,85}$ for the electric field $\mathbf{E}(\mathbf{r})$

$$
\mathbf{E}(\mathbf{r})=\mathbf{V}(\mathbf{r})+\iiint_{\mathrm{R}^{3}} d^{3} \mathbf{r}^{\prime} \underline{\underline{T}}\left(\mathbf{r}-\mathbf{r}^{\prime}\right) \mathbf{P}\left(\mathbf{r}^{\prime}\right),
$$

where $\mathbf{V}(\mathbf{r})$ is the vacuum field (electric field generated by the solute atomic partial charges in the absence of polarizable medium), $\mathbf{P}(\mathbf{r})$ the polarization (dipole moment density), and $\underline{\mathbf{T}}(\mathbf{r})$ the dipole-dipole interaction tensor characterizing the solvent-solvent interactions within the system.

In the application of Eq. (1), it will be assumed that both solute-solute and solute-solvent electrostatic interactions are described by truncated Coulomb interactions with a Barker-Watts reaction-field correction. ${ }^{14-16}$ In the BarkerWatts scheme, the potential generated at $\mathbf{r}$ by a unit charge at the origin is given by

$$
\psi_{\mathrm{BW}}(r)=\frac{1}{4 \pi \epsilon_{o}} H(R-r)\left(r^{-1}+\frac{\alpha r^{2}}{2 R^{3}}-\frac{\alpha+2}{2 R}\right),
$$

where $H(r)$ is the Heaviside function $[H(r)=1$ if $r>0$, $H(r)=0$ otherwise], $\epsilon_{o}$ the permittivity of vacuum, and $R$ is the cut-off distance. The parameter $\alpha$ (with $0 \leqslant \alpha \leqslant 1$ ) determined by the relative dielectric permittivity $\epsilon^{\prime}$ of the medium surrounding the cut-off sphere of each particle through

$$
\alpha=\frac{2\left(\epsilon^{\prime}-1\right)}{2 \epsilon^{\prime}+1} .
$$

The function $\psi_{\mathrm{BW}}$ in Eq. (2) accounts both for the direct Coulombic potential generated by the charge $\left(r^{-1}\right.$ term) and for the polarization by the neighboring charges of the medium outside its cut-off sphere $\left(r^{2}\right.$ term). In the present work, the discussion of the general form of the Barker-Watts interaction function (BW) will essentially focus on the cases $\alpha=0 \quad\left(\epsilon^{\prime}=1\right)$, corresponding to straight truncation of the Coulomb interactions without reaction-field correction (ST), and $\alpha=1\left(\epsilon^{\prime} \rightarrow \infty\right)$, corresponding to truncated Coulomb interactions with a reaction-field correction corresponding to a conducting medium (RF).

When Eq. (2) is applied to the solvent-solvent interactions under nonperiodic boundary conditions (NPBC), the dipole-dipole interaction tensor reads

$$
\begin{aligned}
\underline{\mathbf{T}}_{\mathrm{NPBC}}(\mathbf{r})= & \frac{1}{4 \pi \epsilon_{o}} H\left(R_{\mathrm{SS}}-r\right) \\
& \times \nabla \otimes \nabla\left(r^{-1}+\frac{\alpha r^{2}}{2 R_{\mathrm{SS}}^{3}}-\frac{\alpha+2}{2 R_{\mathrm{SS}}}\right) \\
= & \frac{1}{4 \pi \epsilon_{o}} H\left(R_{\mathrm{SS}}-r\right)\left(\frac{3 \mathbf{r} \otimes \mathbf{r}-r^{2} \underline{\mathbf{1}}}{r^{5}}+\frac{\alpha \underline{\mathbf{1}}}{R_{\mathrm{SS}}^{3}}\right),
\end{aligned}
$$


where $R_{\mathrm{SS}}$ is the solvent-solvent cut-off distance and the notation $\mathbf{a} \otimes \mathbf{b}$ has been introduced for the tensor with elements $\mu \nu$ given by $a_{\mu} b_{\nu}$. Under periodic boundary conditions (PBC), and provided that $R_{\mathrm{SS}}$ is smaller than half the smallest dimension of the computational box (which will be assumed from here on), the dipole-dipole interaction tensor reads

$$
\underline{\mathbf{T}}_{\mathrm{PBC}}(\mathbf{r})=\frac{1}{4 \pi \epsilon_{o}} H\left(R_{\mathrm{SS}}-\bar{r}\right)\left(\frac{3 \overline{\mathbf{r}} \otimes \overline{\mathbf{r}}-\bar{r}^{2} \underline{\mathbf{1}}}{\bar{r}^{5}}+\frac{\alpha \underline{\mathbf{1}}}{R_{\mathrm{SS}}^{3}}\right),
$$

where $\overline{\mathbf{r}}$ is the minimum-image vector corresponding to $\mathbf{r}$.

Using the approximation of linear response, the reaction of the polarizable medium is linear in the local electric field, ${ }^{24,25,51,64,85}$ i.e.,

$$
\mathbf{P}(\mathbf{r})=\epsilon_{o}[\epsilon(\mathbf{r})-1] \mathbf{E}(\mathbf{r}),
$$

where $\epsilon(\mathbf{r})$ the relative dielectric permittivity of the medium, which may be heterogeneous in space. Typically, one distinguishes between solute and solvent regions, characterized by distinct homogeneous permittivity values.

The dipole-dipole interaction tensor $\underline{\mathbf{T}}(\mathbf{r})$ defined by Eqs. (4) or (5) is singular at the origin. However, the singularity is integrable when applying Eq. (1). More precisely, defining $\Omega(\mathbf{r} ; \rho)$ as the sphere of radius $\rho$ and surface $\Sigma(\mathbf{r} ; \rho)$ centered at $\mathbf{r}$, one may write

$$
\begin{aligned}
& \iiint_{\mathbb{R}^{3}} d^{3} \mathbf{r}^{\prime} \underline{\mathbf{T}}\left(\mathbf{r}-\mathbf{r}^{\prime}\right) \mathbf{P}\left(\mathbf{r}^{\prime}\right) \\
& \quad=\mathbf{I}(\mathbf{r})+\lim _{\rho \rightarrow 0} \iiint_{\mathbb{R}^{3} \backslash \Omega(\mathbf{r} ; \rho)} d^{3} \mathbf{r}^{\prime} \underline{\mathbf{T}}\left(\mathbf{r}-\mathbf{r}^{\prime}\right) \mathbf{P}\left(\mathbf{r}^{\prime}\right) .
\end{aligned}
$$

The first term can be evaluated as

$$
\begin{aligned}
\mathbf{I}(\mathbf{r}) & =\lim _{\rho \rightarrow 0} \iiint_{\Omega(\mathbf{r} ; \rho)} d^{3} \mathbf{r}^{\prime} \underline{\mathbf{T}}\left(\mathbf{r}-\mathbf{r}^{\prime}\right) \mathbf{P}\left(\mathbf{r}^{\prime}\right) \\
& =\frac{1}{4 \pi \epsilon_{o}}\left[\lim _{\rho \rightarrow 0} \iiint_{\Omega(\mathbf{0} ; \rho)} d^{3} \mathbf{s} \nabla \otimes \nabla s^{-1}\right] \mathbf{P}(\mathbf{r}) \\
& =-\frac{1}{4 \pi \epsilon_{o}}\left[\lim _{\rho \rightarrow 0} \rho^{-2} \iint_{\Sigma(\mathbf{0} ; \rho)} d^{2} \sigma s^{-2} \mathbf{s} \otimes \mathbf{S}\right] \mathbf{P}(\mathbf{r}) \\
& =-\frac{1}{2 \epsilon_{o}}\left[\int_{0}^{\pi} d \theta \sin \theta \cos ^{2} \theta\right] \mathbf{P}(\mathbf{r})=-\frac{1}{3 \epsilon_{o}} \mathbf{P}(\mathbf{r}) .
\end{aligned}
$$

The second equality follows from inserting Eqs. (4) or (5), defining $\mathbf{s}=\mathbf{r}^{\prime}-\mathbf{r}$, and noting that as $\rho$ tends towards zero: (i) The Heaviside function evaluates to one for any finite $R_{\mathrm{SS}}$; (ii) the contribution proportional to the unit tensor vanishes; (iii) $\mathbf{P}\left(\mathbf{r}^{\prime}\right)$ may be approximated by $\mathbf{P}(\mathbf{r})$ and factorized from the integral. The third equality follows from applying the gradient theorem and inserting $\nabla s^{-1}=-s^{-3} \mathbf{s}$. The fourth equality follows from observing that, due to symmetry, the off-diagonal elements of the tensor vanish upon integration, and that the diagonal elements are all equal. The fifth equality follows from evaluating one of these diagonal elements (integrand $s^{-2} s_{z}^{2}=\cos ^{2} \theta$ ) in spherical coordinates.
Combining Eqs. (1), (6), (7), and (8), the equation to be solved for the electrostatic field $\mathbf{E}(\mathbf{r})$ reads

$$
\begin{aligned}
\mathbf{E}(\mathbf{r})= & \mathbf{V}(\mathbf{r})-\frac{1}{3}[\epsilon(\mathbf{r})-1] \mathbf{E}(\mathbf{r}) \\
& +\epsilon_{o} \lim _{\rho \rightarrow 0} \iiint_{\mathbb{R}^{3} \backslash \Omega(\mathbf{r} ; \rho)} d^{3} \mathbf{r}^{\prime} \underline{\mathbf{T}}\left(\mathbf{r}-\mathbf{r}^{\prime}\right) \\
& \times\left[\epsilon\left(\mathbf{r}^{\prime}\right)-1\right] \mathbf{E}\left(\mathbf{r}^{\prime}\right),
\end{aligned}
$$

with $\underline{\mathbf{T}}(\mathbf{r})$ given by Eqs. (4) or (5). If this equation can be solved for $\mathbf{E}(\mathbf{r})$, the free energy of interaction between the solute atomic point charges and the polarizable medium is given by

$$
\Delta G=-\frac{1}{2} \iiint_{\mathbb{R}^{3}} d^{3} \mathbf{r} \mathbf{V}(\mathbf{r}) \cdot \mathbf{P}(\mathbf{r}) .
$$

In the special case of a nonpolarizable solute, this quantity represents the solvation free energy of the solute.

\section{B. Discretization}

To transform the solving of Eq. (9) into a computationally tractable problem, three approximations are made. First, the infinite integration domain is reduced to a finite region of space. More precisely, two types of computational domains are considered: (i) A spherical volume of radius $S$ surrounded by vacuum under NPBC, or (ii) a cubic unit cell of edge $L$ under PBC. In both cases, the restriction to a finite computational domain is expected to have limited consequences when $S \gg R_{\mathrm{SS}}$ under NPBC or $L \gg 2 R_{\mathrm{SS}}$ under PBC (because the truncation of solvent-solvent interactions largely reduces dipole-dipole correlations at large distances), provided that the vacuum field is only active over a small region within this domain (which will be the case due to truncation of the solute-solvent interactions). Second, the solute is assumed to be nonpolarizable and the solvent to be represented by a medium of homogeneous permittivity. Thus, the computational domain comprises two subdomains characterized by different homogeneous dielectric permittivity values: A (possibly discontinous) solute subdomain of relative permittivity one, and a solvent subdomain of relative permittivity $\epsilon_{s}$. Third, the problem is discretized by paving the computational domain using $N$ grid cells, leading to piecewiseconstant representations $\mathbf{V}_{i}$ and $\mathbf{E}_{i}$ (with $i=1, \ldots, N$ ) of the vacuum and electric fields.

Within these approximations, Eq. (9) becomes

$$
\begin{aligned}
E_{i \mu}= & V_{i \mu}-\frac{\epsilon_{s}-1}{3} \sigma_{i} E_{i \mu} \\
& +\epsilon_{o}\left(\epsilon_{s}-1\right) \sum_{j=1}^{N} v_{j}\left(1-\delta_{i j}\right) \sigma_{j} \sum_{\nu=1}^{3} T_{\mu \nu}\left(\mathbf{r}_{i}-\mathbf{r}_{j}\right) E_{j \nu},
\end{aligned}
$$

where the $\mu$ and $\nu$ indexes enumerate Cartesian components, $\mathbf{r}_{i}$ and $v_{i}$ are the center coordinate and volume of grid cell $i$, the exterior function $\sigma_{i}$ evaluates to one if $\mathbf{r}_{i}$ is within the solvent subdomain and zero otherwise, and the matrix elements of the dipole-dipole interaction tensor [Eqs. (4) or (5)] take the form 


$$
\begin{aligned}
& T_{\mathrm{NPBC}, \mu \nu}(\mathbf{r}) \\
& \quad=\frac{1}{4 \pi \epsilon_{o}} H\left(R_{\mathrm{SS}}-r\right)\left(\frac{3 r_{\mu} r_{\nu}-\delta_{\mu \nu} r^{2}}{r^{5}}+\frac{\alpha \delta_{\mu \nu}}{R_{\mathrm{SS}}^{3}}\right)
\end{aligned}
$$

or

$$
\begin{aligned}
& T_{\mathrm{PBC}, \mu \nu}(\mathbf{r}) \\
& \quad=\frac{1}{4 \pi \epsilon_{o}} H\left(R_{\mathrm{SS}}-\bar{r}\right)\left(\frac{3 \bar{r}_{\mu} \bar{r}_{\nu}-\delta_{\mu \nu} \bar{r}^{2}}{\bar{r}^{5}}+\frac{\alpha \delta_{\mu \nu}}{R_{\mathrm{SS}}^{3}}\right) .
\end{aligned}
$$

Defining the $3 N \times 3 N$-dimensional matrix $\underline{\mathbf{A}}$ as

$$
\begin{aligned}
A_{i \mu j \nu}= & \left(1+\frac{\epsilon_{s}-1}{3} \sigma_{i}\right) \delta_{i j} \delta_{\mu \nu} \\
& -\epsilon_{o}\left(\epsilon_{s}-1\right) v_{j}\left(1-\delta_{i j}\right) \sigma_{j} T_{\mu \nu}\left(\mathbf{r}_{i}-\mathbf{r}_{j}\right),
\end{aligned}
$$

Eq. (11) can be rewritten in matrix notation

$$
\underline{\mathbf{A}} \mathbf{E}=\mathbf{V} \text {. }
$$

Because the size of the matrix $\underline{\mathbf{A}}$ is generally very large, it cannot be stored in memory and direct methods cannot be used to solve Eq. (15). Therefore, an under-relaxed Jacobi method is applied here to obtain successive approximate solutions for the discretized electric field. Given the approximate solution $\mathbf{E}^{(k)}$ at iteration $k$, the grid $\mathbf{E}^{(k+1)}$ at the next iteration is computed as

$$
\mathbf{E}^{(k+1)}=\mathbf{E}^{(k)}-\lambda \underline{\mathbf{D}}^{-1}\left(\underline{\mathbf{A}} \mathbf{E}^{(k)}-\mathbf{V}\right),
$$

where $\mathbf{D}$ is the diagonal matrix defined by the diagonal elements of $\underline{\mathbf{A}}$, and $\lambda$ (with $0<\lambda \leqslant 1$ ) is a relaxation parameter. A reasonable initial guess for $\mathbf{E}^{(0)}$ is provided by the vacuum field scaled by the solvent permittivity, i.e.,

$$
\mathbf{E}^{(0)}=\epsilon_{s}^{-1} \mathbf{V} .
$$

It is easily seen that a self-consistent solution of Eq. (16) must satisfy Eq. (15). In order to assess the convergence of the numerical solution upon iterating, the residual (with units of an electric field)

$$
\tau^{(k)}=\left(\frac{\sum_{i=1}^{N} v_{i} \sigma_{i}\left\|\left(\underline{\mathbf{A}} \mathbf{E}^{(k)}-\mathbf{V}\right)_{i}\right\|^{2}}{\sum_{i=1}^{N} v_{i} \sigma_{i}}\right)^{1 / 2},
$$

is introduced as a measure of accuracy.

After solving Eq. (15) for the discretized electric field, the solvation free energy can be evaluated as [Eq. (10); nonpolarizable solute]

$$
\Delta G_{\mathrm{solv}}=-\frac{1}{2} \epsilon_{o}\left(\epsilon_{s}-1\right) \sum_{i=1}^{N} v_{i} \sigma_{i} \mathbf{V}_{i} \cdot \mathbf{E}_{i},
$$

where Eq. (6) was used. Note that the solvation free energy solely depends on the electric field within the solvent subdomain. Furthermore, due to the form of Eq. (14), the field $\mathbf{E}_{i}$ corresponding to a point $i$ in the solvent subdomain does not depend on the field $\mathbf{E}_{j}$ at any point $j$ within the solute subdomain. For this reason, increased computational efficiency can be achieved by omitting all grid points of the solute subdomain from the definition of the matrix $\underline{\mathbf{A}}$ and the determination of the solution of Eq. (15).

\section{Application to solvated spherical ions}

For a system consisting of a single spherical ion of radius $R_{I}$ and charge $q_{I}$ centered in the computational domain, the vacuum field corresponding to ion-solvent interactions described by the Barker-Watts scheme [Eq. (2)] is given by

$$
\mathbf{V}(\mathbf{r})=\frac{q_{I}}{4 \pi \epsilon_{o}} H\left(R_{\mathrm{IS}}-r\right)\left(\frac{\mathbf{r}}{r^{3}}-\frac{\alpha \mathbf{r}}{R_{\mathrm{IS}}^{3}}\right),
$$

where $R_{\mathrm{IS}}$ is the ion-solvent cut-off distance. This equation is valid under both NPBC and PBC, provided that $R_{\mathrm{IS}}$ is smaller than half the smallest dimension of the computational box (which will be assumed from here on).

Because the ion is generally small compared to the size of the computational domain, while the variations of the electric field within the solvent are typically largest close to its surface, the accuracy of the results will depend crucially on the detailed representation of the ionic surface. For this reason, two levels of grid resolution are used. First, a coarse grid of spacing $\Delta$ is generated, that covers the entire computational domain (leading to grid-cell volumes $v_{i}=\Delta^{3}$ ). Second, all cells of the coarse grid with their center closer than $(\sqrt{3} / 2) \Delta$ from the surface of the ion are further discretized, i.e., they are replaced by a set of finer grid cells of edge $\delta$ $=n^{-1} \Delta$ where $n$ is a positive integer (leading to grid-cell volumes $v_{i}=\delta^{3}$ ). Any grid-cell center of the finer grid that is closer than $R_{I}$ from the ion center is discarded from the calculation (solute point). In this representation, the vacuum potential at any grid point $i$ is evaluated in practice as

$$
\mathbf{V}_{i}=\frac{q_{I}}{4 \pi \epsilon_{o}} I\left(R_{\mathrm{IS}}-r_{i}\right)\left(\frac{\mathbf{r}_{i}}{r_{i}^{3}}-\frac{\alpha \mathbf{r}_{i}}{R_{\mathrm{IS}}^{3}}\right),
$$

where $I\left(R_{\mathrm{IS}}-r_{i}\right)$ represents the fraction of the grid cell located within the ion-solvent cut-off sphere. ${ }^{64}$

After solving Eq. (15) for the discretized electric field, the radial polarization $p(r)$ around the ion [Eq. (6)] can be computed in the form of a histogram. To avoid artifacts linked with the use of two different grid spacings, this calculation is based on a uniform grid of $N^{\prime}$ points obtained by partitioning all cells of the coarse grid into finer grid cells of edge $\delta$ sharing a common value of the electic field. Under $\mathrm{NPBC}$, the radial polarization is then computed as

$$
\begin{aligned}
p_{\mathrm{NPBC}}\left(r_{n}\right)= & \epsilon_{o}\left(\epsilon_{s}-1\right) H\left(r_{n}-R_{I}\right) \\
& \times \frac{\sum_{i=1}^{N^{\prime}} \sigma_{i} w\left(r_{i} ; r_{n}, \Delta r\right) r_{i}^{-1} \mathbf{r}_{i} \cdot \mathbf{E}_{i}}{\sum_{i=1}^{N^{\prime}} \sigma_{i} w\left(r_{i} ; r_{n}, \Delta r\right)},
\end{aligned}
$$

where

$$
r_{n}=\left(n+\frac{1}{2}\right) \Delta r, \quad n=0,1, \ldots, n_{\max },
$$

$\Delta r$ being the histogram width, and

$$
w\left(r ; r_{n}, \Delta r\right)= \begin{cases}1 & \text { if } r_{n}-\frac{1}{2} \Delta r \leqslant r<r_{n}+\frac{1}{2} \Delta r \\ 0 & \text { otherwise. }\end{cases}
$$

Under PBC, the periodic copies of the central box must be taken into account and Eq. (22) is modified to 


$$
\begin{aligned}
p_{\mathrm{PBC}}\left(r_{n}\right)= & \epsilon_{o}\left(\epsilon_{s}-1\right) H\left(r_{n}-R_{I}\right) \\
& \times \frac{\sum_{\mathbf{l} \in \mathbb{Z}^{3}} \sum_{i=1}^{N^{\prime}} \sigma_{i} w\left(r_{i, \mathrm{l}} ; r_{n}, \Delta r\right) r_{i, \mathbf{1}}^{-1} \mathbf{r}_{i, \mathbf{I}} \cdot \mathbf{E}_{i}}{\sum_{\mathbf{l} \in \mathbb{Z}^{3}} \sum_{i=1}^{N^{\prime}} \sigma_{i} w\left(r_{i, 1} ; r_{n}, \Delta r\right)},
\end{aligned}
$$

with $\mathbf{r}_{i, \mathbf{l}}=\mathbf{r}_{i}+L \mathbf{l}$. In practice, the sum over $\mathbf{l}$ is restricted to vectors with integer components in the range $\left[-l_{\max } ; l_{\max }\right]$, ensuring a correct description of the polarization up to $r$ $=\left(l_{\max }+1 / 2\right) L$. These polarization histograms can be compared to the ideal (CB/NPBC) Born polarization ${ }^{86}$

$$
p_{\text {Born }}(r)=\frac{q_{I}}{4 \pi} \frac{\epsilon_{s}-1}{\epsilon_{s}} r^{-2} .
$$

Combining Eqs. (19) and (21), the ionic solvation free energy is evaluated (based on the refined grid of $N^{\prime}$ points) as

$$
\begin{aligned}
\Delta G_{\mathrm{solv}}= & -\frac{q_{I}}{8 \pi}\left(\epsilon_{s}-1\right) \sum_{i=1}^{N^{\prime}} v_{i} \sigma_{i} I\left(R_{\mathrm{IS}}-r_{i}\right) \\
& \times\left(\frac{\mathbf{r}_{i}}{r_{i}^{3}}-\frac{\alpha \mathbf{r}_{i}}{R_{\mathrm{IS}}^{3}}\right) \cdot \mathbf{E}_{i} .
\end{aligned}
$$

This value can be compared to the ideal (CB/NPBC) Born electrostatic solvation free energy ${ }^{86}$

$$
\Delta G_{\mathrm{solv}}^{\mathrm{Born}}=-\frac{q_{I}^{2}}{8 \pi \epsilon_{o}} \frac{\epsilon_{s}-1}{\epsilon_{s}} R_{I}^{-1} .
$$

The application to two (or more) spherical ions is straightforward and only requires the following minor adaptations: (i) The quantity $\sigma_{i}$ is zero (point discarded from the calculation) for all grid cells with centers located inside any ion, and one otherwise; (ii) the vacuum field $\mathbf{V}$ [Eq. (21)] is expressed as a sum of contributions arising from each ion; (iii) the solvation free energy $\Delta G_{\text {solv }}[\mathrm{Eq} .(27)]$ is expressed as a sum of contributions arising from each ion; (iv) fine grids (spacing $\delta<\Delta$ ) are used to describe the close neighborhood of all ions.

\section{COMPUTATIONAL DETAILS}

The solution of Eq. (15), restricted to the case of one or two spherical ions, was implemented in a $\mathrm{C}$ program. The single ion or the two ions are placed on the $z$ axis of the coordinate system (single ion at $z=0$; two ions at $z=$ $\pm d / 2$, where $d$ is the interionic distance). Taking advantage of the symmetry of the problem, the storage of the discretized vacuum and electric fields is only required for one quadrant $(x, y \geqslant 0)$ of the computational domain.

After an evaluation of the convergence properties of the algorithm, a set of computational parameters was selected and adopted for all subsequent calculations. The corresponding values are as follows (unless otherwise specified). The spacings corresponding to the coarse and fine grids were set to $\Delta=0.1 \mathrm{~nm}$ and $\delta=0.025 \mathrm{~nm}$, respectively. The relaxation parameter $\lambda$ was set to 0.4 . The algorithm was terminated when the residual $\tau^{(k)}$ was either below $10^{-3} \mathrm{~kJ} \mathrm{~mol}^{-1}$ $\mathrm{nm}^{-1} e^{-1}$ or reached a minimum value. All calculations un- der NPBC used a sphere of radius $S=4.0 \mathrm{~nm}$ as computational domain. To ensure that this domain is large enough to be representative of an infinite nonperiodic system, a number of single-ion and two-ion calculations were repeated with $S$ $=5.0 \mathrm{~nm}$. The observed differences in solvation free energy were in all cases below $0.1 \mathrm{~kJ} \mathrm{~mol}^{-1}$ in magnitude.

The method was first applied to solvated spherical ions. Ionic solvation free energies [Eq. (27)] and radial polarization histograms [Eqs. (22) or (25) with $\Delta r=0.025 \mathrm{~nm}$ and $\left.l_{\max }=2\right]$ were computed for all combinations of the following parameters: ionic charge $q_{I}=1 e$, ionic radii $R_{I}$ $=0.2 \mathrm{~nm}$ (about the size of $\mathrm{Na}^{+}$) or $0.4 \mathrm{~nm}$ (about the size of $\mathrm{Cl}^{-}$), cut-off radii $R_{\mathrm{IS}}=R_{\mathrm{SS}}=R_{C}=0.8$ or $1.2 \mathrm{~nm}$, solvent permittivities $\epsilon_{s}=2$ (alkanelike solvent) or 78 (water), $\alpha$ $=0$ (ST scheme) or 1 (RF scheme), and NPBC or PBC (with $L=2.6 \mathrm{~nm}$ ). To validate the method, the results are compared with those of calculations employing other methods (Table I), namely: (i) 1D-Direct ${ }^{27,28}$ (NPBC; bin size 0.005 $\mathrm{nm}$, range $4.0 \mathrm{~nm}$ ) or (ii) $3 \mathrm{D}-\mathrm{FFT}^{60}$ (PBC; 180 grid points along each Cartesian axis).

The method was then used to investigate the effect of periodicity on ionic solvation free energies in systems with truncated electrostatic interactions (ST or RF). To this end, ionic solvation free energies were computed under PBC using the above combination of parameters, for cubic box edges $L$ ranging from $1.6 \mathrm{~nm}\left(R_{C}=0.8 \mathrm{~nm}\right)$ or $2.4 \mathrm{~nm}\left(R_{C}\right.$ $=1.2 \mathrm{~nm}$ ) to $8.0 \mathrm{~nm}$. The effect on periodicity can be quantified by the relative periodicity-induced perturbation of the ionic solvation free energy $\gamma(L)$, defined as

$$
\gamma(L)=\frac{\Delta \Delta G_{\mathrm{solv}}(L)}{\Delta G_{\mathrm{solv}}^{\mathrm{NPC}}}
$$

with

$$
\Delta \Delta G_{\mathrm{solv}}(L)=\Delta G_{\mathrm{solv}}^{\mathrm{PBC}}(L)-\Delta G_{\mathrm{solv}}^{\mathrm{NPC}} .
$$

Finally, the effect of cut-off truncation (ST or RF) and periodicity on the electrostatic solvation contribution $\Delta G_{\text {solv }}(d)$ to the potential of mean force for the interaction between two ions at distance $d$ (under PBC, ions aligned along an axis of the cubic unit cell) was evaluated for the special case of charges $q_{I}= \pm q_{J}=1 \quad e$, radii $R_{I}=R_{J}$ $=0.4 \mathrm{~nm}$, cut-off radii $R_{\mathrm{IS}}=R_{\mathrm{SS}}=R_{C}=1.2 \mathrm{~nm}$ and for a cubic unit-cell of edge $L=6 \mathrm{~nm}$ (PBC). For validation, the results under PBC were compared with those of calculations employing the $3 \mathrm{D}-\mathrm{FFT}^{60}$ method (180 grid points along each Cartesian axis). The corresponding overall electrostatic contribution $\Delta G_{\mathrm{el}}(d)$ to the potential of mean force was also evaluated as

$$
\Delta G_{\mathrm{el}}(d)=\Delta G_{\mathrm{solv}}(d)+q_{I} q_{J} \psi_{\mathrm{BW}}(\widetilde{d})
$$

where $\widetilde{d}=d(\mathrm{NPBC})$ or $\widetilde{d}=\min \{d ; L-d\}(\mathrm{PBC})$, and $\psi_{\mathrm{BW}}$ is given by Eq. (2) with $\alpha=0$ (ST) or $\alpha=1$ (RF). These profiles can be compared with the expected long-range behavior of the electrostatic potential of mean force for a Coulombic interaction between the ions, namely 

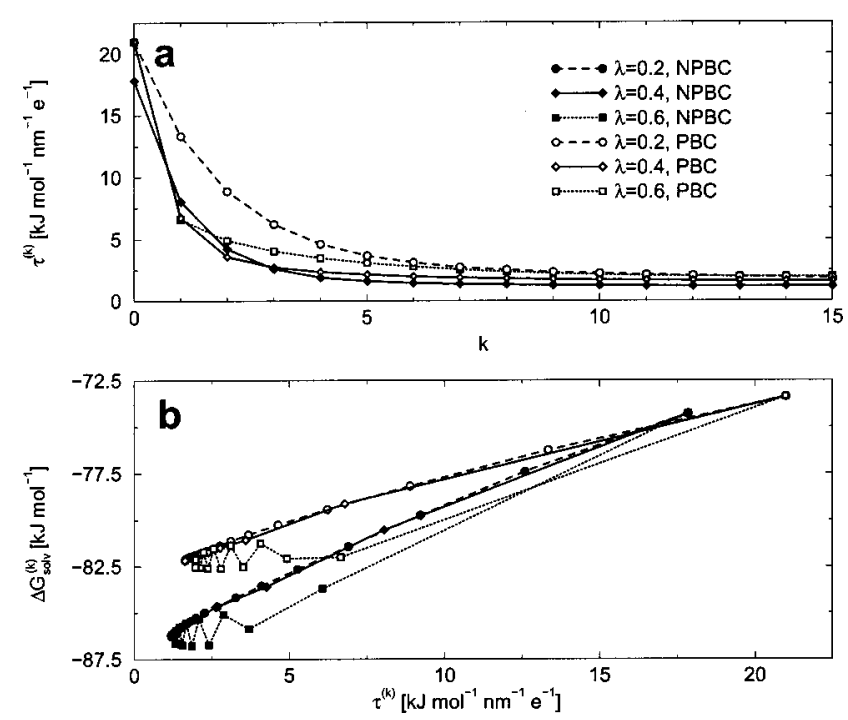

FIG. 2. Convergence properties of the under-relaxed Jacobi algorithm [Eq. (16)] used to solve Eq. (15). The residual $\tau^{(k)}$ [Eq. (18)] is displayed as a function of the number of iterations $k$ (a), and the solvation free energy $\Delta G_{\text {solv }}^{(k)}$ at iteration $k$ [Eq. (27)] as a function of the corresponding residual $\tau^{(k)}$ (b). The system consists of a single spherical ion of charge $q_{I}=1 e$ and radius $R_{I}=0.4 \mathrm{~nm}$ in a solvent of permittivity $\epsilon_{s}=78$, and is either nonperiodic (NPBC; spherical domain of radius $S=4.0 \mathrm{~nm}$ ) or periodic (PBC; cubic unit cell of edge $L=2.6 \mathrm{~nm}$ ). Electrostatic interactions correspond to the RF scheme, with cut-off radii $R_{\mathrm{IS}}=R_{\mathrm{SS}}=R_{C}=1.2 \mathrm{~nm}$. Three choices of the Jacobi relaxation parameter $\lambda$ are compared [for NPBC, only the curve corresponding to $\lambda=0.4$ is displayed in (a)].

$$
\begin{aligned}
\Delta G_{\mathrm{el}}^{l r}(d)= & \Delta G_{\mathrm{solv}}^{\mathrm{ion}}\left(q_{I}, R_{I}\right)+\Delta G_{\mathrm{solv}}^{\mathrm{ion}}\left(q_{J}, R_{J}\right) \\
& +\frac{q_{I} q_{J}}{4 \pi \epsilon_{o} \epsilon_{s}}, d^{-1}
\end{aligned}
$$

where $\Delta G_{\text {solv }}^{\text {ion }}(q, R)$ is the solvation free energy of an isolated ion of radius $R$ and charge $q$ under NPBC when applying the specific electrostatic scheme.

\section{RESULTS}

\section{A. Convergence properties}

The convergence properties of the under-relaxed Jacobi algorithm [Eq. (16)] used to solve Eq. (15) are illustrated in Fig. 2 for a spherical ion of charge $q_{I}=1 e$ and radius $R_{I}$ $=0.4 \mathrm{~nm}$ in a solvent of permittivity $\epsilon_{s}=78$, based on the RF scheme with a single cut-off radius $R_{C}=1.2 \mathrm{~nm}$ and using three choices of the relaxation parameter $\lambda$. Results for the ST scheme are qualitatively very similar (data not shown). Within few iterations, the residual $\tau^{(k)}$ decreases from about 20 to values below $3 \mathrm{~kJ} \mathrm{~mol}^{-1} \mathrm{~nm}^{-1} e^{-1}$ [Fig. 2(a)]. Convergence to zero residual only occurs when the solvent permittivity $\epsilon_{s}$ is smaller than about 10 (data not shown). This limited convergence is probably related to the presence of a strong discontinuity in the system permittivity at the ion-solvent boundary in the case of high solvent permittivities. For $\boldsymbol{\epsilon}_{s}$ values larger than about 10, the residual reaches a minimum after a certain number of iterations (typically about $15-20$ for $\lambda=0.4$ ) and slowly rises again afterwards. When this situation occurred, the algorithm was terminated at the minimum value $\tau$ of the residual. However, convergence of $\tau^{(k)}$ towards $\tau$ is associated with the simultaneous convergence of $\Delta G_{\text {solv }}^{(k)}$ to a well-defined value $\Delta G_{\text {solv }}$ [Fig. 2(b) and Table II]. Because the values of both the minimum residual and the associated converged solvation free energy are essentially independent of the convergence parameter $\lambda$, it appears that the method is nevertheless able to produce accurate results for high $\epsilon_{s}$ values. This is also supported by the observation that for $\epsilon_{s}=2$, values of $\Delta G_{\text {solv }}^{(k)}$ when $\tau^{(k)}=3 \mathrm{~kJ} \mathrm{~mol}^{-1} \mathrm{~nm}^{-1} e^{-1}$ typically differ from the corresponding converged values $\left(\tau^{(k)}\right.$ $<10^{-3} \mathrm{~kJ} \mathrm{~mol}^{-1} \mathrm{~nm}^{-1} e^{-1}$ ) by less than $1 \%$ (data not shown).

Although the convergence parameter $\lambda$ does not influence the final values of $\tau$ and $\Delta G_{\text {solv }}$, it has a strong impact on the convergence rate. For $\epsilon_{s}=78, \lambda=0.2$ leads to slow convergence, $\lambda=0.6$ to slow convergence and oscillatory evolution of $\Delta G_{\text {solv }}^{(k)}$, while the algorithm fails to converge for $\lambda=0.8$ (data not shown). In practice, it was found that $\lambda=0.4$ is the optimal choice in this case, and is also adequate for $\epsilon_{s}=2$ (although a somewhat larger values may slightly accelerate convergence). This value was adopted for all subsequent calculations.

The rates of convergence to the minimum $\tau$ of $\tau^{(k)}$ appear to be very similar under NPBC or PBC, and for the RF and ST (data not shown) schemes. The final values of the residual, however, are somewhat lower under NPBC compared to PBC (Table II).

\section{B. Single spherical ion}

The radial polarization $p(r)$ around around a spherical ion [Eqs. (22) and (25)] of charge $q_{I}=1 e$ and radius $R_{I}$ $=0.4 \mathrm{~nm}$ in a solvent of permittivity $\epsilon_{s}=78$ is displayed in Fig. 3 for different choices of boundary conditions and treatments of the electrostatic interactions based on a single cutoff $R_{C}=1.2 \mathrm{~nm}$. The polarization corresponding to the Born model $^{86}[\mathrm{CB} / \mathrm{NPBC}$; Eq. (26)] or to the lattice-sum case (LS/ PBC; computed using the 3D-FFT method ${ }^{64}$ ), and the polarization computed from the 1D-Direct method ${ }^{27,28}$ (ST,RF/ NPBC) are also displayed for comparison. For both the ST and RF schemes under NPBC, the agreement between the results of the 1D-Direct and of the present 3D-Direct methods is excellent over the whole range of distances. The only noticeable difference is the more progressive transition of $p(r)$ around $R_{C}$ in the 3D-Direct calculation for the ST scheme, which is due to a significantly lower resolution and to the smoothing of the vacuum field at the ion-solvent cutoff distance [function $I$ in Eq. (21)]. These curves, however, differ significantly from the Born polarization, corresponding to the ideal situation of a spherical ion solvated by a nonperiodic Coulombic continuum of infinite extent.

For the ST case, the polarization curve is discontinuous at the ion-solvent cut-off distance $R_{\mathrm{IS}}$. The polarization below $R_{\mathrm{IS}}$ is consistently larger than predicted by the Born model, whereas the polarization above is smaller, although always positive. Underpolarization of the solvent above $R_{\mathrm{IS}}$ is easily understood since the solvent beyond this distance does not feel directly the electrostatic field of the ion. However, the solvent in this region reacts indirectly to the ionic 
TABLE II. Solvation free energy $\Delta G_{\text {solv }}$ of a single spherical ion [Eq. (27)] computed using the 3D-Direct method (present article). The system consists of a single ion of charge $q_{I}=1 e$ and radius $R_{I}$ in a solvent of permittivity $\epsilon_{s}$, and is either nonperiodic (NPBC; spherical domain of radius $S=4 \mathrm{~nm}$ ) or periodic (PBC; cubic unit cell of edge $L=2.6 \mathrm{~nm}$ ). Electrostatic interactions correspond to either the ST or RF schemes, with cut-off radii $R_{\mathrm{IS}}=R_{\mathrm{SS}}=R_{C}$. For the $\mathrm{RF}$ scheme, the reaction-field contribution to the solvation free energy [ $\alpha$-dependent term in Eq. (27)] is reported between parentheses. The converged value $\tau$ of the residual is also indicated. The solvation free energies $\Delta G_{\text {solv }}^{\text {corr }}$ corrected by the inclusion of a self-energy term [Eq. (B3)] are also given. For comparison, the corresponding Born solvation free energies [Eq. (28)] are $-342.9\left(\epsilon=78, R_{I}\right.$ $=0.2 \mathrm{~nm}),-171.4\left(\epsilon=78, R_{I}=0.4 \mathrm{~nm}\right),-173.7\left(\epsilon=2, R_{I}=0.2 \mathrm{~nm}\right)$, and $-86.8\left(\epsilon=2, R_{I}=0.4 \mathrm{~nm}\right)$ $\mathrm{kJ} \mathrm{mol}^{-1}$.

\begin{tabular}{|c|c|c|c|c|c|c|c|c|}
\hline $\mathrm{BC}$ & Interaction & $\epsilon_{s}$ & $\begin{array}{c}R_{I} \\
{[\mathrm{~nm}]}\end{array}$ & $\begin{array}{c}R_{C} \\
{[\mathrm{~nm}]}\end{array}$ & {$\left[\mathrm{kJ} \mathrm{mol}^{-1} \mathrm{~nm}^{-1} e^{-1}\right]$} & $\begin{array}{c}\Delta G_{\mathrm{solv}} \\
{\left[\mathrm{kJ} \mathrm{mol}^{-1}\right]}\end{array}$ & $\begin{array}{c}\Delta G_{\mathrm{solv}}^{\mathrm{ref}} \\
{\left[\mathrm{kJ} \mathrm{mol}^{-1}\right]}\end{array}$ & $\begin{array}{c}\Delta G_{\text {solv }}^{\text {corr }} \\
{\left[\mathrm{kJ} \mathrm{mol}^{-1}\right]}\end{array}$ \\
\hline NPBC & ST & 78 & 0.2 & 0.8 & 1.2 & -284.8 & $-281.7^{\mathrm{a}}$ & -370.5 \\
\hline NPBC & ST & 78 & 0.2 & 1.2 & 1.2 & -306.3 & $-303.4^{\mathrm{a}}$ & -363.4 \\
\hline NPBC & ST & 78 & 0.4 & 0.8 & 0.8 & -100.9 & $-100.1^{\mathrm{a}}$ & -186.6 \\
\hline NPBC & ST & 78 & 0.4 & 1.2 & 0.7 & -129.4 & $-128.8^{\mathrm{a}}$ & -186.5 \\
\hline PBC & ST & 78 & 0.2 & 0.8 & 3.0 & -284.1 & $-280.3^{b}$ & -369.8 \\
\hline $\mathrm{PBC}$ & ST & 78 & 0.2 & 1.2 & 2.9 & -301.0 & $-297.9^{\mathrm{b}}$ & -355.0 \\
\hline $\mathrm{PBC}$ & ST & 78 & 0.4 & 0.8 & 2.0 & -100.6 & $-102.4^{b}$ & -186.3 \\
\hline $\mathrm{PBC}$ & ST & 78 & 0.4 & 1.2 & 1.8 & -125.3 & $-125.7^{b}$ & -182.4 \\
\hline NPBC & RF & 78 & 0.2 & 0.8 & 1.1 & $-217.0(37.0)$ & $-213.7(37.1)^{\mathrm{a}}$ & -345.6 \\
\hline NPBC & RF & 78 & 0.2 & 1.2 & 1.1 & $-259.2(26.2)$ & $-256.3(26.4)^{\mathrm{a}}$ & -344.9 \\
\hline NPBC & $\mathrm{RF}$ & 78 & 0.4 & 0.8 & 0.6 & $-47.6(23.7)$ & $-46.8(23.7)^{\mathrm{a}}$ & -176.2 \\
\hline NPBC & $\mathrm{RF}$ & 78 & 0.4 & 1.2 & 0.6 & $-86.4(22.0)$ & $-85.8(22.2)^{\mathrm{a}}$ & -172.1 \\
\hline PBC & $\mathrm{RF}$ & 78 & 0.2 & 0.8 & 2.8 & $-214.6(35.8)$ & $-211.0(35.6)^{b}$ & -343.2 \\
\hline $\mathrm{PBC}$ & $\mathrm{RF}$ & 78 & 0.2 & 1.2 & 2.9 & $-253.1(22.3)$ & $-249.5(22.5)^{b}$ & -338.8 \\
\hline $\mathrm{PBC}$ & RF & 78 & 0.4 & 0.8 & 1.6 & $-47.5(22.9)$ & $-48.4(23.0)^{\mathrm{b}}$ & -176.1 \\
\hline $\mathrm{PBC}$ & RF & 78 & 0.4 & 1.2 & 1.6 & $-82.3(18.7)$ & $-82.3(18.8)^{\mathrm{b}}$ & -168.0 \\
\hline NPBC & ST & 2 & 0.2 & 0.8 & 0.0 & -133.4 & $-135.2^{\mathrm{a}}$ & -176.8 \\
\hline NPBC & ST & 2 & 0.2 & 1.2 & 0.0 & -146.7 & $-148.3^{\mathrm{a}}$ & -175.6 \\
\hline NPBC & ST & 2 & 0.4 & 0.8 & 0.0 & -45.6 & $-46.4^{\mathrm{a}}$ & -89.0 \\
\hline NPBC & ST & 2 & 0.4 & 1.2 & 0.0 & -60.3 & $-60.8^{\mathrm{a}}$ & -89.2 \\
\hline $\mathrm{PBC}$ & ST & 2 & 0.2 & 0.8 & 0.0 & -133.1 & $-135.0^{\mathrm{b}}$ & -176.5 \\
\hline $\mathrm{PBC}$ & ST & 2 & 0.2 & 1.2 & 0.0 & -145.6 & $-147.6^{b}$ & -174.5 \\
\hline $\mathrm{PBC}$ & ST & 2 & 0.4 & 0.8 & 0.0 & -45.6 & $-46.3^{\mathrm{b}}$ & -89.0 \\
\hline $\mathrm{PBC}$ & ST & 2 & 0.4 & 1.2 & 0.0 & -59.6 & $-60.2^{\mathrm{b}}$ & -88.5 \\
\hline NPBC & RF & 2 & 0.2 & 0.8 & 0.0 & $-99.8(13.7)$ & $-101.0(13.8)^{\mathrm{a}}$ & -164.9 \\
\hline NPBC & RF & 2 & 0.2 & 1.2 & 0.0 & $-123.1(9.8)$ & $-124.4(9.8)^{\mathrm{a}}$ & -166.5 \\
\hline NPBC & $\mathrm{RF}$ & 2 & 0.4 & 0.8 & 0.0 & $-20.4(9.0)$ & $-20.6(9.1)^{\mathrm{a}}$ & -85.5 \\
\hline NPBC & $\mathrm{RF}$ & 2 & 0.4 & 1.2 & 0.0 & $-39.2(8.3)$ & $-39.5(8.4)^{\mathrm{a}}$ & -82.6 \\
\hline $\mathrm{PBC}$ & $\mathrm{RF}$ & 2 & 0.2 & 0.8 & 0.0 & $-99.5(13.8)$ & $-100.9(13.8)^{b}$ & -164.6 \\
\hline $\mathrm{PBC}$ & $\mathrm{RF}$ & 2 & 0.2 & 1.2 & 0.0 & $-122.4(9.5)$ & $-124.0(9.5)^{\mathrm{b}}$ & -165.8 \\
\hline $\mathrm{PBC}$ & $\mathrm{RF}$ & 2 & 0.4 & 0.8 & 0.0 & $-20.4(9.0)$ & $-20.7(9.1)^{b}$ & -85.5 \\
\hline $\mathrm{PBC}$ & $\mathrm{RF}$ & 2 & 0.4 & 1.2 & 0.0 & $-38.9(8.0)$ & $-39.2(8.1)^{b}$ & -82.3 \\
\hline
\end{tabular}

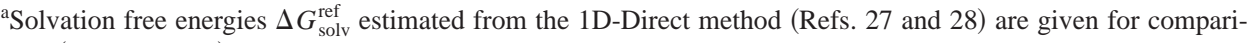
son (ST,RF/NPBC).

${ }^{\mathrm{b}}$ Solvation free energies $\Delta G_{\text {solv }}^{\text {ref }}$ estimated from the 3D-FFT method (Ref. 60) are given for comparison (ST,RF/PBC).

field through interactions with the polarized solvent within the cut-off sphere of the ion, leading to the observed residual polarization. Inside the cut-off sphere of the ion, the solvent is overpolarized because each solvent volume element only interacts with a fraction of the highly polarized solvent within the cut-off sphere of the ion. This partial interaction results in a bias of the solvent polarization towards the ion.

For the RF case, the polarization curve is continuous at $R_{\mathrm{IS}}$, although its derivative is not. The polarization is consistently smaller than the corresponding Born curve over the whole range of distance, both below and above $R_{\mathrm{IS}}$. The difference between the curves is largest at distances close to $R_{\mathrm{IS}}$, and becomes progressively smaller at either short or long distances from the ion. It has been shown that in the limit of high solvent permittivities, the Barker-Watts poten- tial represents (for the solvent-solvent interactions) the cutoff-truncated polynomial of second order (terms in $r^{-1}$ to $r^{2}$ ) that leads to the best agreement between Born and effective polarizations. ${ }^{27,28} \mathrm{~A}$ number of additional results related to this comparison are derived in Appendix A, namely that (i) the RF/NPBC polarization converges to the Born polarization in the limit $R_{\mathrm{IS}}, R_{\mathrm{SS}} \rightarrow \infty$; (ii) in the limit of small distances (compared to $R_{C}$ ), the RF/NPBC polarization converges towards the Born polarization; (iii) in the limit of large distances (compared to $R_{C}$ ), the RF/NPBC polarization becomes proportional to $r^{-2}$, just as the Born polarization.

For both the ST and RF schemes, the polarization curves corresponding to $\mathrm{PBC}$ are systematically lower (in the range $R_{I}$ to $L$ ) compared to the polarization under NPBC. The reason for this is that under $\mathrm{PBC}$, the solvent in the reference 

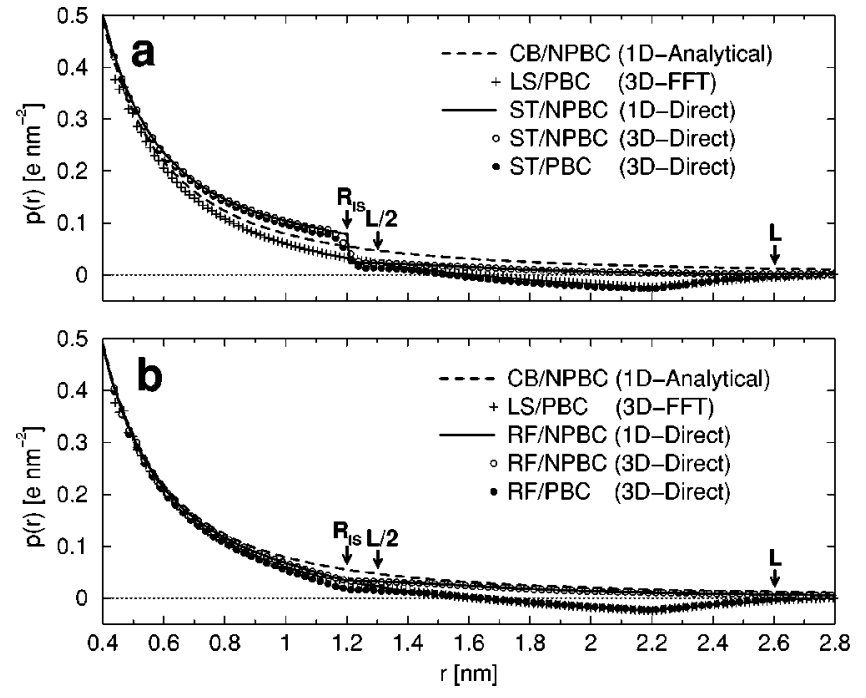

FIG. 3. Radial polarization $p(r)$ around a solvated spherical ion [Eqs. (22) or (25)]. The system consists of a single ion of charge $q_{I}=1 e$ and radius $R_{I}=0.4 \mathrm{~nm}$ in a solvent of permittivity $\epsilon_{s}=78$, and is either nonperiodic (NPBC; spherical domain of radius $S=4.0 \mathrm{~nm}$ ) or periodic (PBC; cubic unit cell of edge $L=2.6 \mathrm{~nm}$ ). Electrostatic interactions correspond to either the $\mathrm{ST}$ (a) or RF (b) schemes, with cut-off radii $R_{\mathrm{IS}}=R_{\mathrm{SS}}=R_{C}=1.2 \mathrm{~nm}$. In addition to the results of the 3D-Direct method (present article), the analytical polarization corresponding to the Born model [CB/NPBC; Eq. (26)] and the lattice-sum case [LS/PBC; computed using the 3D-FFT method (Ref. 64)], and the polarization computed from the 1D-Direct method (Refs. 27 and 28) for the specific interaction scheme (ST,RF/NPBC) are also presented for comparison. The cut-off distance as well as the (half-)box edge (PBC only) are indicated by arrows.

unit cell is perturbed by its interaction with the solvent in adjacent unit cells (itself polarized by the periodic copies of the ion) and, for $r>L-R_{\mathrm{IS}}$, with the periodic copies of the ion themselves. The consequences of these interactions are a depolarization of the solvent in the reference unit cell (compared to NPBC), and the occurence of negative polarization values for the solvent in the neighboring unit cells. At large distances, $p(r)$ displays an irregular oscillatory behavior with values close to zero at the location of the nearest neighbor ions (i.e., $L, \sqrt{2} L, \sqrt{3} L, \ldots$; data not shown). The depolarization of the solvent within the reference unit cell is slightly more important in the RF case compared to the ST case, which is probably a consequence of the larger magnitude of the residual solvent polarization above $R_{\mathrm{IS}}$ observed in the RF/NPBC case. In both cases, however, the solvent depolarization within the reference unit cell remains relatively small because, due to the truncation of ion-solvent interactions at $R_{\mathrm{IS}}<L / 2$, dipoles in the reference unit cell do not interact directly with the periodic copies of the ion. Finally, the polarization corresponding to the RF/PBC scheme is seen to agree reasonably well with the LS/PBC curve, the difference being expectedly largest in the neighborhood of the cut-off distance. There is, however, an important difference between the two schemes. When cut-off truncation is applied, the solvation free energy only depends on the polarization in the range $R_{I}$ to $R_{\mathrm{IS}}<L / 2$ [Eqs. (10) and (20)] and the effect of periodicity on the ionic solvation free energy is expected to be relatively small. If this restriction is removed (e.g., when nontruncated LS interactions are considered), the effect of artificial periodicity on the ionic solvation free en- ergy becomes dramatically more important. ${ }^{19,55,57}$

Ionic solvation free energies $\Delta G_{\text {solv }}$ computed for spherical ions with different parameter combinations, electrostatic schemes, and boundary conditions are listed in Table II. The values $\Delta G_{\text {solv }}^{\text {ref }}$ computed using the 1D-Direct method $^{27,28}$ (ST,RF/NPBC) or the 3D-FFT method ${ }^{60}$ (ST,RF/ $\mathrm{PBC}$ ) are also listed for comparison. Note that, while the former values are certainly very accurate, the latter values are probably subject to errors of a similar magnitude as the present method. The agreement between the values computed using different methods is in general very good. The average and maximal relative differences between the present 3D-Direct and the reference values are $1.1 \%$ and $1.9 \%$, respectively. Not unexpectedly, these relative differences tend to be somewhat larger for (i) the smaller ionic radius, (ii) the larger permittivity value, (iii) periodic boundary conditions. The following observations can be made: (i) the solvation free energies are larger in magnitude for the smaller ion and the larger permittivity value, in qualitative agreement with the Born model; (ii) the solvation free energies are larger in magnitude for the larger cut-off value, a consequence of including a larger amount of polarized solvent within the cut-off sphere of the ion; (iii) the solvation free energies are more negative for the ST scheme compared to the RF scheme, a consequence of the solvent overpolarization within the cut-off sphere of the ion for the ST scheme (Fig. 3) and of the inclusion of an additional positive term in the solvation free energy for the RF scheme $[\alpha$-dependent term in Eq. (27); reported in Table II between parentheses]; (iv) the solvation free energies are larger in magnitude under NPBC compared to PBC, a consequence of the periodicityinduced solvent depolarization within the reference unit cell (Fig. 3); (v) the periodicity-induced perturbation of the solvation free energy $(\mathrm{NPBC} \rightarrow \mathrm{PBC})$ is larger for the smaller ion, for the larger cut off, for the higher permittivity value, and for RF compared to ST. The latter effect is a consequence of the larger periodicity-induced solvent depolarization within the reference unit cell for the RF scheme (Fig. 3).

The values reported in Table II are strongly cut-offdependent and compare poorly with the corresponding Born solvation free energies of -342.9 and $-171.4 \mathrm{~kJ} \mathrm{~mol}^{-1}$ $\left(\epsilon_{s}=78, q_{I}=1 e, R_{I}=0.2\right.$ or $\left.0.4 \mathrm{~nm}\right)$ or -173.7 and $-86.8 \mathrm{~kJ} \mathrm{~mol}^{-1}\left(\epsilon_{s}=2, q_{I}=1 e, R_{I}=0.2\right.$ or $\left.0.4 \mathrm{~nm}\right)$. As discussed in Appendix B, these large discrepancies could be reduced by the inclusion of a charge self-energy term into the total electrostatic energy of the system. It is also suggested that such a self-energy term should be systematically included in the total electrostatic energy during molecular simulations relying on effective cut-off-based electrostatic interaction functions to ensure the obtension of meaningful energies. In this context, a new definition [Eq. (B4)] is proposed for the electrostatic interaction energy in simulations employing the Barker-Watts reaction-field scheme.

The effect of artificial periodicity on the solvation free energy of a spherical ion computed using cut-off-based (ST or RF) electrostatics is illustrated in Fig. 4 for an ion of charge $q_{I}=1 e$, a solvent of permittivity $\epsilon_{s}=78$, and for different values of the ionic radius $R_{I}$ and cut-off radius $R_{C}$. The relative periodicity-induced perturbation $\gamma(L)$ of the 

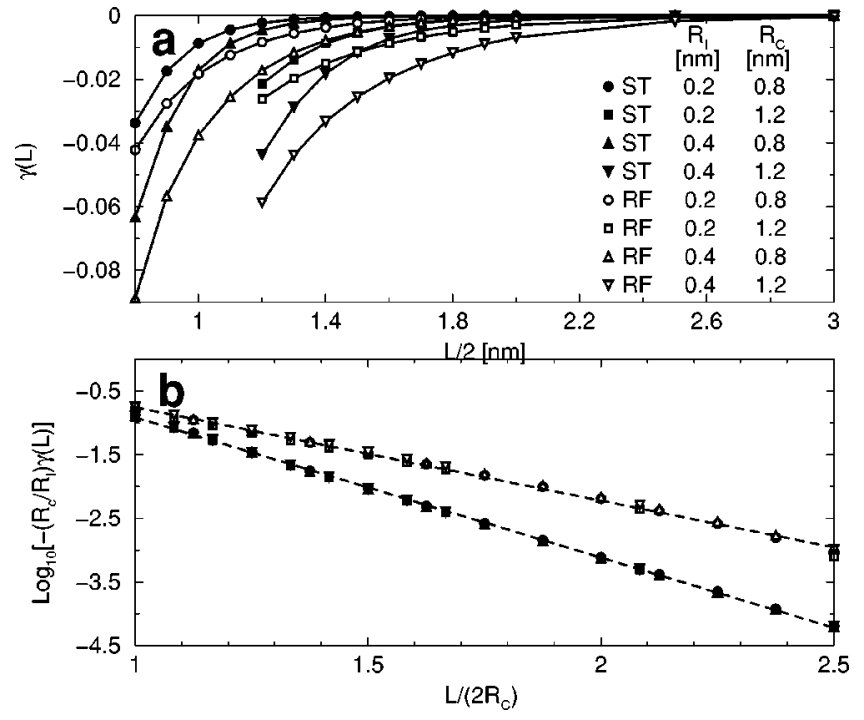

FIG. 4. Periodicity-induced perturbation of the solvation free energy of a spherical ion. The system consists of a single ion of charge $q_{I}=1 e$ and radius $R_{I}$ in a cubic periodic box of edge $L$ filled by a solvent of permittivity $\epsilon_{s}=78$. Electrostatic interactions correspond to either the ST or RF schemes, with cut-off radii $R_{\mathrm{IS}}=R_{\mathrm{SS}}=R_{C}$. (a) Relative periodicity-induced shift $\gamma(L)$ in the solvation free energy [Eq. (29)], displayed as a function of $L / 2$. (b) Logarithm of minus $\gamma(L)$ amplified by $R_{C} / R_{I}$, displayed as a function of $L / 2 R_{C}$. The dashed lines corresponding to a least-squares fit [over the interval $L /\left(2 R_{C}\right)=0-2.5$ ] corresponding to either the RF or the ST schemes.

ionic solvation free energy [Eq. (29)] is displayed as a function of $L / 2$ in Fig. 4(a). All curves converge to a limiting value of one when $L / 2 \gg R_{C}$, indicating that the solvation free energy under PBC indeed converges to its NPBC value when the computational box becomes large compared to the cut-off radius. For example, the magnitude of $\gamma(L)$ for $L / 2$ $=4 \mathrm{~nm}$ is smaller than $10^{-4}$ for all parameter combinations considered. When $L / 2$ is only moderately larger than $R_{C}$, artificial periodicity causes a depolarization of the solvent (Fig. 3) and a decrease in the magnitude of the solvation free energy. As a consequence, $\gamma(L)$ becomes negative. When $L / 2=R_{C}$, the solvation free energy is reduced by $2 \%-9 \%$ compared to its NPBC value for the parameter combinations considered. In agreement with previous observations (Table II), the relative periodicity-induced perturbation of the solvation free energy (i) increases in magnitude with increasing ionic radius; (ii) increases in magnitude with increasing cutoff radius; (iii) is larger for the RF scheme compared to the ST scheme.

The different curves in Fig. 4(a) can be adequately represented by exponential functions. This is shown in Fig. 4(b), where the quantity $\log _{10}\left[-\left(R_{C} / R_{I}\right) \gamma(L)\right]$ is displayed as a function of $L /\left(2 R_{C}\right)$. The resulting data can be fitted by two straight lines, corresponding to the ST and RF schemes, with linear correlation coefficients (over the interval $L /\left(2 R_{C}\right)$ $=0$ to 2.5$)$ of -0.9998 and -0.9985 , respectively. Thus, irrespective of $R_{I}$ and $R_{C}$, the relative periodicity-induced perturbation appears to be approximately of the form

$$
\gamma(L) \approx-\frac{R_{I}}{R_{C}} 10^{\mu L /\left(2 R_{C}\right)+\nu} .
$$

Based on all available data for $\varepsilon_{s}=78$, the constants in Eq. (32) evaluated to $\mu=-2.203$ and $\nu=1.290$ for the ST scheme, and $\mu=-1.468$ and $\nu=0.715$ for the RF scheme.

This behavior can be contrasted to the case of nontruncated electrostatic interactions. In this case, the solvation free energy corresponding to $\mathrm{CB} / \mathrm{NPBC}$ is given by the Born expression $^{86}$ [Eq. (28)]. A corresponding analytical expression ${ }^{57}$ has been derived for the LS/PBC case, namely

$$
\begin{aligned}
\Delta G_{\mathrm{solv}}^{\mathrm{LS} / \mathrm{PBC}}= & -\frac{q_{I}^{2}}{8 \pi \epsilon_{o}} \frac{\epsilon_{s}-1}{\epsilon_{s}}\left[\frac{L}{R_{I}}+\xi_{\mathrm{EW}}\right. \\
& \left.+\frac{4 \pi}{3}\left(\frac{R_{I}}{L}\right)^{2}-\frac{16 \pi^{2}}{45}\left(\frac{R_{I}}{L}\right)^{5}\right] L^{-1},
\end{aligned}
$$

with $\xi_{\mathrm{EW}} \approx-2.837297$. Thus, it follows from Eq. (29) that:

$$
\gamma(L)=\frac{R_{I}}{L}\left[\xi_{\mathrm{EW}}+\frac{4 \pi}{3}\left(\frac{R_{I}}{L}\right)^{2}-\frac{16 \pi^{2}}{45}\left(\frac{R_{I}}{L}\right)^{5}\right] .
$$

In this case, the evolution of $\gamma(L)$ towards zero when $L$ $\gg R_{I}$ is in $L^{-1}$, i.e., much slower than the exponential distance-dependence observed for cut-off-based schemes [Eq. (32)]. For example, for an ion of radius $R_{I}=0.4 \mathrm{~nm}$, $\gamma(L)$ evaluates to -0.95 for $L / 2=0.4 \mathrm{~nm},-0.19$ for $L / 2$ $=3.0 \mathrm{~nm}$, and is above -0.1 for $L / 2>5.7 \mathrm{~nm}$ [compare with the smaller magnitude and faster relaxation observed for cut-off-based schemes in Fig. 4(a)]. This shows that the application of a cut-off in the computation of ionic solvation free energies by explicit-solvent simulation dramatically reduces the system-size dependence of the calculated solvation free energies compared to lattice-sum methods. ${ }^{19,55,57}$ More generally, cut-off truncation (with the possible inclusion of a reaction-field correction) efficiently reduces the impact of finite-size effects and artificial periodicity on the energies and forces in any molecular dynamics simulation. However, this is at the expense of introducing other (potentially more harmful) artifacts related with the cut-off truncation itself.

\section{Interaction between two spherical ions}

The electrostatic solvation free energy profiles $\Delta G_{\text {solv }}(d)$ for a pair of monovalent spherical ions (same or opposite charges, identical radii of $0.4 \mathrm{~nm}$ ) in a solvent of permittivity $\epsilon_{s}=78$ are displayed in Fig. 5 as a function of the interionic distance $d$ for different choices of boundary conditions and treatments of the electrostatic interactions based on a single cut-off $R_{C}=1.2 \mathrm{~nm}$. The PBC curves correspond to ions aligned along an axis of a cubic unit cell of edge $L=6 \mathrm{~nm}$. The corresponding profiles computed using the 3D-FFT method ${ }^{60}$ (ST,RF/PBC) are also displayed for comparison.

As expected, the curves corresponding to the NPBC case present a minimum (maximum) at ionic contact for ions of identical (opposite) charges, and asymptotically converge to a common value for a given scheme. More precisely, in the limit of large interionic distances (isolated ions), $\Delta G_{\text {solv }}(d)$ converges towards twice the solvation free energy $\Delta G_{\text {solv }}^{\text {ion }}$ of a single ion $\left(\Delta G_{\text {solv }}^{\text {ion }}=-129.4\right.$ or $-86.4 \mathrm{~kJ} \mathrm{~mol}^{-1}$ for the ST or RF schemes; see Table II). In the limit $d \rightarrow 0$ (superim- 

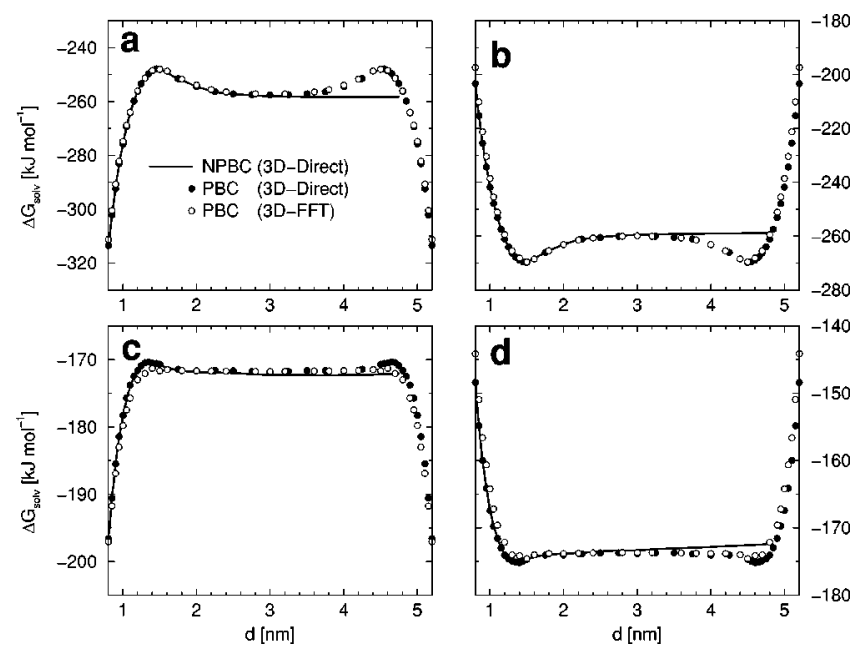

FIG. 5. Electrostatic solvation free energy profile $\Delta G_{\text {solv }}(d)$ for a pair of monovalent spherical ions. The system consists of two ions of radii $R_{I}$ $=0.4 \mathrm{~nm}$ bearing identical ( $\mathrm{a}$ and $\mathrm{c}$ ) or opposite (b and d) charges, and separated by a distance $d$ in a medium of permittivity $\epsilon_{s}=78$. It is either nonperiodic (NPBC; spherical domain of radius $S=4.0 \mathrm{~nm}$ ) or periodic (PBC; cubic unit cell of edge $L=6 \mathrm{~nm}$; ions aligned with an axis of the unit cell). Electrostatic interactions correspond to either the ST (a and b) or RF (c and d) schemes, with cut-off radii $R_{\mathrm{IS}}=R_{\mathrm{SS}}=R_{C}=1.2 \mathrm{~nm}$. In addition to the results of the 3D-Direct method (present article), the solvation free energies computed from the 3D-FFT method (Refs. 51 and 60) for the specific interaction scheme (ST,RF/PBC) and the same value of $L$ are also presented for comparison.

posed ions), $\Delta G_{\text {solv }}(d)$ converges towards four times $\Delta G_{\text {solv }}^{\text {ion }}$ (zero) for ions of identical (opposite) charges.

Under PBC, symmetry and periodicity constraints impose that the profile possesses a stationary point at $d=L / 2$ and is symmetrical with respect to this point. This induces a difference between the NPBC and PBC curves for $d$ in the interval $[0 ; L / 2]$. Within this range, the perturbation caused by the introduction of periodicity is attractive (repulsive) for ions of identical (opposite) charges. However, the magnitude of the effect is very small. For example, the differences between the values of $\Delta G_{\text {solv }}(L / 2)$ under NPBC and PBC is only about $0.45 \mathrm{~kJ} \mathrm{~mol}^{-1}$ in magnitude for all cases considered. Thus, in contrast to the case of lattice-sum methods, ${ }^{57}$ artificial periodicity has very little influence on the solvation free energy profile for pairs of small monovalents ions in a solvent of high permittivity when cut-off truncation is applied. Finally, the agreement between the present calculations employing the 3D-Direct method under PBC and the 3D-FFT method ${ }^{60}$ is quite good, especially for the ST scheme. For the RF scheme, the agreement is slightly worse, probably due to small differences in the application of boundary smoothing at the ion surface and ion-solvent cutoff distance. ${ }^{60}$

The corresponding profiles for the overall electrostatic contribution $\Delta G_{\mathrm{el}}(d)$ to the potential of mean force [Eq. (30)] are displayed in Fig. 6 as a function of the interionic distance $d$. At short distances, the curves corresponding to NPBC and PBC are nearly identical. In the ST case, the curves present minima (maxima) at contact and at the cut-off distance for ions of identical (opposite) charges. The presence of an extremum at the cut-off distance is clearly an
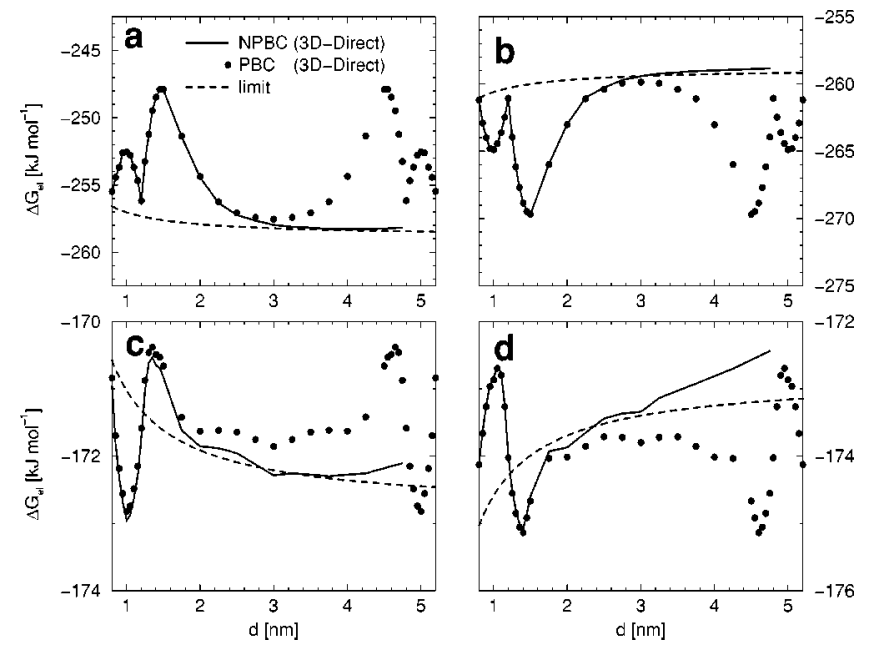

FIG. 6. Electrostatic contribution $\Delta G_{\mathrm{el}}(d)$ to the potential of mean force for a pair of monovalent spherical ions. The system consists of two ions of radii $R_{I}=0.4 \mathrm{~nm}$ bearing identical (a and c) or opposite ( $\mathrm{b}$ and $\mathrm{d}$ ) charges, and separated by a distance $d$ in a medium of permittivity $\epsilon_{s}=78$. It is either nonperiodic (NPBC; spherical domain of radius $S=4.0 \mathrm{~nm}$ ) or periodic (PBC; cubic unit cell of edge $L=6 \mathrm{~nm}$; ions aligned with an axis of the unit cell). Electrostatic interactions correspond to either the ST (a and b) or RF (c and d) schemes, with cut-off radii $R_{\mathrm{IS}}=R_{\mathrm{SS}}=R_{C}=1.2 \mathrm{~nm}$. The ideal longrange limit $\Delta G_{\mathrm{el}}^{\mathrm{lr}}(d)$ is also presented for comparison [Eq. (31)]. It is calculated using $\Delta G_{\text {solv }}^{\text {ion }}=-129.4(\mathrm{ST})$ or $-86.4(\mathrm{RF}) \mathrm{kJ} \mathrm{mol}^{-1}$ (Table II).

artifact related to the use of truncated electrostatic interactions. These profiles provide an explanation for a number of observations made in explicit-solvent simulations employing the ST scheme: (i) The tendency for ion pairs with like charges to cluster at distances close to the cut-off distance, ${ }^{9,96}$ (ii) the tendency for ion pairs of opposite charges to avoid distances close to the cut-off distance ${ }^{9,42,43,96}$ (iii) the artificially increased ${ }^{38,40,41,57}$ stability of contact ion pairs for ions of like charges; ${ }^{36,37,39,41,87}$ (iv) the artificially decreased stability of contact pairs for ions of opposite charges. ${ }^{42,44,80}$

In the RF case, the profiles nearly present the expected behavior, namely repulsion (attraction) for ions of like (opposite) charges, except for a significant artifact in the neighborhood of the cut-off distance. For ions of like charges, a spurious minimum occurs just below the cut-off distances, while for ions of opposite charges, a spurious maximum occurs just above the cut-off distance. Although these artifacts might affect the populations of contact pairs for ions of like or opposite charges in simulations of ionic solution, ${ }^{88,89}$ the magnitude of these artifacts is limited compared to the ST scheme, in agreement with previous observations. ${ }^{44,90}$

At large distances from the ion, and for both the ST and RF schemes, the NPBC profiles tend to be close to the expected Coulombic limit [Eq. (31)]. However, the exact agreement is difficult to assess since positive deviations occur, which are probably related to the limited size of the computational domain.

\section{CONCLUSION}

In the present study, continuum electrostatics was used to investigate the nature and magnitude of the perturbations induced by cut-off truncation and artificial periodicity in 
explicit-solvent simulations of ions and ion pairs. To this purpose, a new algorithm relying on finite integration was developed to solve the equations of continuum electrostatics based on truncated (and possibly reaction-field corrected) solute-solvent and solvent-solvent electrostatic interactions, under either nonperiodic (NPBC) or periodic (PBC) boundary conditions. This algorithm was tested and validated by comparison with available methods (Table I) whenever possible.

In the context of the solvation of a single spherical ion, the main observations can be summarized as follows:

(A) The application of cut-off truncation (under NPBC) significantly affects the solvent polarization around a spherical ion (compared to the ideal Born result). With straight truncation (ST) of the interactions, the solvent is overpolarized within the cut-off sphere of the ion and underpolarized outside this sphere. When a reactionfield $(\mathrm{RF})$ correction is applied, the agreement with the Born (NPBC) or lattice-sum (PBC) polarization is significantly improved, the deviations being largest in the neighborhood of the cut-off distance.

(B) The introduction of artificial periodicity (NPBC $\rightarrow$ PBC) when applying cut-off-based electrostatics leads to a depolarization of the solvent around the ion in the reference unit cell. This effect is caused by the indirect (solvent-mediated) perturbation of the solvent molecules in this reference cell by the periodic copies of the ion. The depolarization is more significant for the RF scheme compared to the ST scheme.

(C) The application of cut-off truncation (under NPBC) decreases the magnitude of the ionic solvation free energy of a spherical ion (compared to the ideal Born result). The magnitude of this effect is highly sensitive to the electrostatic scheme (ST or RF) and to the choice of a cut-off radius. However, as discussed in Appendix $\mathrm{B}$, the problem could be largely (though approximately) remedied in explicit-solvent simulations by the systematic inclusion of an appropriate self-energy term in the total electrostatic energy of the system. Alternatively, an exact correction term to ionic solvation free energies computed from explicit-solvent simulations can be obtained by the application of the present continuum-electrostatics method under NPBC or of its one-dimensional analog. ${ }^{27,28}$

(D) The introduction of artificial periodicity (NPBC $\rightarrow$ PBC) when applying cut-off-based electrostatics causes a further decrease in the magnitude of the ionic solvation free energy. In contrast to lattice-sum methods, ${ }^{57}$ where this free-energy shift is important even for relatively large system sizes (proportional to $L^{-1}, L$ being the edge length of the cubic unit cell), the effect decays rapidly with increasing system sizes (proportional to $R^{-1} \exp (-c L / R), R$ being the cut-off distance) in the case of cut-off-based electrostatics. Here also, an approximate correction term was derived [Eq. (34)] that can be applied to correct ionic solvation free energies evaluated from explicit-solvent simulations under PBC.
The relevance of these observations can be appreciated by recalling the five sources of error related to the computation of ionic solvation free energies from explicit-solvent simulations relying on cut-off-based electrostatic interactions: (i) Incorrect polarization of the solvent around the ion due to truncated (and possibly modified) electrostatic interactions; (ii) Cut-off- and system size-dependent perturbation of the solvent polarization due to artificial periodic boundary conditions; (iii) artifacts at the cut-off distance arising from the finite size of the solvent molecules, and related to the use of either a molecular or an atomic cut-off; (iv) artificial heating during molecular-dynamics simulations due to the possible presence of discontinuities in the atomic forces; (v) inaccuracy of the ion-solvent and solvent-solvent interaction functions and parameters (force fields).

Only with the understanding of these five sources of error and the design of appropriate correction schemes will it be possible to obtain accurate ionic solvation free energies from explicit-solvent simulations. The discussion (and correction) of the two first sources of error has been the focus of the present article. The third problem has been previously discussed by a number of groups. ${ }^{55,72-75,77,78,91}$ Due to the finite size of solvent molecules, the solvent-generated potential at the ion site (and thus the solvation free energy) may vary considerably depending on whether cut-off truncation is applied on an atomic or on a molecular basis (and in the latter case, on the choice of a molecular center). Although the debate is not yet completely settled, a number of arguments suggest that molecular truncation (based on the center of van der Waals interactions for a spherical molecule ${ }^{77}$ ) represents the appropriate method for evaluating the solventgenerated potential at the ion site, ${ }^{74,77,91}$ while a (generally sizeable) correction term must be applied if atomic truncation (or a lattice-sum method) is employed instead. The fourth problem, namely the artificial heating of molecules at distances close to the cut-off radius, may be alleviated by the use of an effective truncated electrostatic interaction that is continuously differentiable at the cut-off distance, together with atomic truncation. For example, the Barker-Watts reaction-field interaction ${ }^{14-16}$ causes very limited heating provided that the permittivity of the solvent considered is high and that an (unusual) atomic-cut-off implementation is applied. ${ }^{16}$ Finally, in regard to the fifth problem, it should be stressed that the derivation of force-field parameters for ionsolvent interactions based on experimental ionic solvation free energies makes little sense before the four other problems are solved (i.e., methodology-independent ionic solvation free energies can be be obtained from explicit-solvent simulations).

In the context of the potential of mean force for the interaction between two spherical ions, the main observations can be summarized as follows:

(A) The application of cut-off truncation (under NPBC) induces serious artifacts in the overall electrostatic contribution to the potential of mean force for the interaction between two spherical ions. As previously observed in explicit-solvent simulations, these lead to spurious features in the radial distribution functions 
close to the cut-off distance $9,42,43$ and to artifacts in the relative stability of the contact, solvent-separated and free ion pairs. ${ }^{36-42,44,57,80,87}$ These effects are reduced (although not compleletly eliminated) by the application of a reaction-field correction.

(B) The introduction of artificial periodicity (NPBC $\rightarrow$ PBC) when applying cut-off-based electrostatics appears to cause very small changes in the electrostatic contribution to the (minimum-image) potentials of mean force for small monovalent ions in a solvent of high permittivity. A rather weak periodicity-induced perturbation was also reported in this case for latticesum methods. ${ }^{57}$ However, the causes of the limited overall effect are different. In the cut-off case, both the solvation free energy profile and the direct ion-ion interaction are almost unaffected by periodicity. In the lattice-sum case, both contributions are largely affected, but the two perturbations nearly cancel each other.

Explicit-solvent simulations of ion solvation and ionion interactions are currently in progress to confirm the validity of the above considerations derived from a continuumelectrostatics analysis, and their compatibility with the results of simulations employing lattice-sum methods.

\section{ACKNOWLEDGMENTS}

The Swiss National Science Foundation (Project 2100061939) is gratefully acknowledged for financial support, as well as Peter Gee for his careful reading of the manuscript and helpful suggestions.

\section{APPENDIX A: POLARIZATION IN THE BARKER-WATTS REACTION-FIELD SCHEME}

As shown in Fig. 3, the polarization corresponding to the RF scheme under NPBC is very close to the corresponding Born polarization, the deviation being largest at distances close to the cut-off radius. Here, we derive a number of results related to this comparison, in the more general context of the Barker-Watts (BW) interaction function [Eq. (2)], namely that (i) the BW/NPBC and CB/NPBC (Born) polarizations become identical in the limit $R_{\mathrm{IS}}, R_{\mathrm{SS}} \rightarrow \infty$, irrespective of the value of $\alpha$; (ii) when $R_{\mathrm{IS}}=R_{\mathrm{SS}}$, the BW/NPBC polarization converges towards the Born polarization at short distances from the ion, provided that $\alpha=2\left(\epsilon_{s}-1\right) /\left(2 \epsilon_{s}\right.$ $+1)$; (iii) the BW/NPBC polarization, just as the Born polarization, is proportional to $r^{2}$ at large distances from the ion, provided that $\alpha=\left(\epsilon_{s}+2\right) /\left(\epsilon_{s}-1\right)$. The latter results obviously remain approximately valid for a solvent of high permittivity $\left(\epsilon_{s} \gg 1\right)$ when $\alpha$ is set to one (RF) or close to one.

The derivations are based on continuum-electrostatics results presented in a previous article, ${ }^{27,28}$ and applied to the specific case of truncated electrostatic interactions corresponding to the Barker-Watts potential [Eq. (2)]. In this case the radial polarization around a solvated spherical ion is a solution of the integral equation

$$
\begin{aligned}
p(r)= & g(r)+\frac{\epsilon_{s}-1}{\epsilon_{S}} \int_{\max \left(R_{I},\left|r-R_{\mathrm{SS}}\right|\right)}^{r+R_{\mathrm{SS}}} K\left(r, r^{\prime}\right) p\left(r^{\prime}\right) d r^{\prime} \\
& \text { for } r>R_{I},
\end{aligned}
$$

together with $p(r)=0$ for $r \leqslant R_{I}$. The inhomogeneous term $g(r)$ is related to the vacuum field generated by the ion

$$
g(r)=\frac{q_{I}}{4 \pi} \frac{\epsilon_{s}-1}{\epsilon_{s}} H\left(R_{\mathrm{IS}}-r\right)\left(r^{-2}-\frac{\alpha r}{R_{\mathrm{IS}}^{3}}\right),
$$

and the kernel $K\left(r, r^{\prime}\right)$ to the form of the solvent-solvent interactions

$K\left(r, r^{\prime}\right)=-(\alpha+2) \frac{r^{4}+\left[R_{\mathrm{SS}}^{2}-\left(r^{\prime}\right)^{2}\right]^{2}-2 r^{2}\left[R_{\mathrm{SS}}^{2}+\left(r^{\prime}\right)^{2}\right]}{16 r^{2} R_{\mathrm{SS}}^{3}}$.

First, it is shown that in the limit $R_{\mathrm{IS}}, R_{\mathrm{SS}} \rightarrow \infty$, the polarization defined by Eq. (A1) converges to the Born polarization [Eq. (26)]. Due to the form of Eq. (A2), $\lim _{R_{\mathrm{IS}} \rightarrow \infty}\left[g(r)-p_{\mathrm{Born}}(r)\right]=0$. It is thus sufficient to prove that the integral term in Eq. (A1) vanishes in the limit of an infinite solvent-solvent cut-off radius. In this limit, the lower bound of integration can be set to $R_{\mathrm{SS}}-r$. For $r^{\prime}$ within the interval $R_{\mathrm{SS}}-r$ to $R_{\mathrm{SS}}+r, K\left(r, r^{\prime}\right)$ is positive and possesses a single maximum at $\widetilde{r}^{\prime}=\left(R_{\mathrm{SS}}^{2}+r^{2}\right)^{1 / 2}$ with $K\left(r, \widetilde{r}^{\prime}\right)=(\alpha$ $+2) /\left(4 R_{\mathrm{SS}}\right)$. Assuming that the polarization is positive and finite over the whole range of distance (with a maximum value $\widetilde{p}$ ), upper and lower bounds can be given to the integral in Eq. (A1)

$$
\begin{aligned}
0 & \leqslant \int_{R_{\mathrm{SS}}-r}^{R_{\mathrm{SS}}+r} K\left(r, r^{\prime}\right) p\left(r^{\prime}\right) d r^{\prime} \\
& \leqslant 2 r \tilde{p} K\left(r, \widetilde{r}^{\prime}\right)=\frac{\alpha+2}{2} \widetilde{p} \frac{r}{R_{\mathrm{SS}}},
\end{aligned}
$$

which shows that the integral vanishes for any finite $r$ in the limit $R_{\mathrm{SS}} \rightarrow \infty$. Thus, the $\mathrm{BW} / \mathrm{NPBC}$ polarization converges to the Born polarization in the limit of large cut-off radii, irrespective of the value of $\alpha$. This result is in particular valid for the $\mathrm{ST}^{27}(\alpha=0)$ and $\mathrm{RF}(\alpha=1)$ schemes.

As a second result, it is shown that the polarization defined by Eq. (A1) converges to the Born polarization in the limit of short distances when $R_{\mathrm{IS}}=R_{\mathrm{SS}}$, provided that $\alpha$ $=2\left(\epsilon_{s}-1\right) /\left(2 \epsilon_{s}+1\right)$, i.e., when Eq. (3) is used with $\epsilon^{\prime}$ $=\epsilon_{s}$ (adjusted boundary conditions ${ }^{61}$ ). For $r \leqslant \min \left[R_{\mathrm{IS}}, R_{\mathrm{SS}}\right.$ $\left.-R_{I}\right]$, the Heaviside function in Eq. (A2) can be omitted and the lower bound in Eq. (A1) replaced by $R_{\mathrm{SS}}-r$. Thus, one looks for a solution of

$$
\begin{aligned}
p(r)= & \frac{\epsilon_{s}-1}{\epsilon_{S}}\left[\frac{q_{I}}{4 \pi}\left(r^{-2}-\frac{\alpha r}{R_{\mathrm{IS}}^{3}}\right)\right. \\
& \left.+\int_{R_{\mathrm{SS}}-r}^{R_{\mathrm{SS}}+r} K\left(r, r^{\prime}\right) p\left(r^{\prime}\right) d r^{\prime}\right] .
\end{aligned}
$$

Using the result

$$
\int_{R_{\mathrm{SS}}-r}^{R_{\mathrm{SS}}+r} K\left(r, r^{\prime}\right)\left(r^{\prime}\right)^{-2} d r^{\prime}=\frac{\alpha+2}{3} \frac{r}{R_{\mathrm{SS}}^{3}},
$$




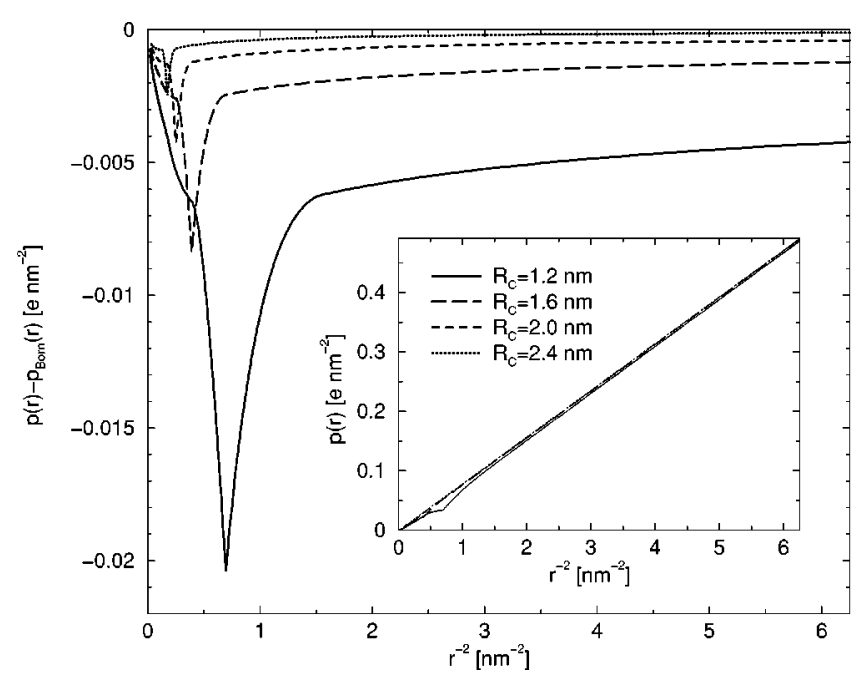

FIG. 7. Radial polarization $p(r)$ around a solvated spherical ion [Eqs. (22)] compared to the Born polarization [Eq. (26)]. The system consists of a single ion of charge $q_{I}=1 e$ and radius $R_{I}=0.4 \mathrm{~nm}$ in a solvent of permittivity $\epsilon_{s}=78$ under NPBC (spherical domain of radius $S=10.0 \mathrm{~nm}$ ). Electrostatic interactions correspond to the RF scheme, with cut-off radii $R_{\mathrm{IS}}$ $=R_{\mathrm{SS}}=R_{C}=1.2,1.6,2.0$, or $2.4 \mathrm{~nm}$. The polarization is computed using the 1D-Direct method (Refs. 27 and 28) for the specific interaction scheme (RF/NPBC). In the inset, $p(r)$ is displayed as a function of $r^{-2}$, while the Born polarization $p_{\mathrm{Born}}(r)$ (not displayed) is a straight line corresponding to the diagonal of the graph. In the main graph, the difference $p(r)$ $-p_{\text {Born }}(r)$ is displayed as a function of $r^{-2}$.

one shows easily that the function satisfying Eq. (A5) when $R_{\mathrm{IS}}=R_{\mathrm{SS}}$ and $\alpha=2\left(\epsilon_{s}-1\right) /\left(2 \epsilon_{s}+1\right)$ is the Born polarization [Eq. (26)]. Therefore, with this specific value of $\alpha$, the BW/NPBC polarization will converge to the Born polarization at short distances, irrespective of the cut-off values. For a solvent of high permittivity $\left(\epsilon_{s} \gg 1\right)$, this result will remain approximately valid when $\alpha=1$ (RF).

As a third result, it is shown that the polarization defined by Eq. (A1) possesses an $r^{-2}$ dependence in the limit of large distances (just as the Born polarization), provided that $\alpha=\left(\epsilon_{s}+2\right) /\left(\epsilon_{s}-1\right)$. For $r \geqslant \max \left[R_{\mathrm{IS}}, R_{\mathrm{SS}}+R_{I}\right]$, Eq. (A2) implies $g(r)=0$ and the lower bound in Eq. (A1) can be replaced by $r-R_{\mathrm{SS}}$. Thus, one looks for a solution of

$$
p(r)=\frac{\epsilon_{s}-1}{\epsilon_{s}} \int_{r-R_{\mathrm{SS}}}^{r+R_{\mathrm{SS}}} K\left(r, r^{\prime}\right) p\left(r^{\prime}\right) d r^{\prime} .
$$

Using the result

$$
\int_{r-R_{\mathrm{SS}}}^{r+R_{\mathrm{SS}}} K\left(r, r^{\prime}\right)\left(r^{\prime}\right)^{-2} d r^{\prime}=\frac{\alpha+2}{3} r^{-2},
$$

one shows easily that the function satisfying Eq. (A7) when $\alpha=\left(\epsilon_{s}+2\right) /\left(\epsilon_{s}-1\right)$ is $c r^{-2}$, where $c$ is a constant. Therefore, with this specific value of $\alpha$, the BW/NPBC polarization will possess a $r^{-2}$ dependence in the limit of large distances. For solvent of high permittivities $\left(\epsilon_{s} \gg 1\right)$, this result will remain approximately valid when $\alpha=1$ (RF) or when $\alpha$ is given by Eq. (3) with $\epsilon^{\prime}=\epsilon_{s}$ (adjusted boundary conditions).

The above observations are illustrated in Fig. 7 for the case of an ion of radius $R_{I}=0.4 \mathrm{~nm}$ immersed in a solvent of permittivity $\epsilon_{s}=78$. In the inset, the polarization $p(r)$ is dis- played as a function of $r^{-2}$ for the RF scheme ( $\left.\alpha=1\right)$, using four different values of the cut-off radius $R_{C}$. The Born polarization is linear in $r^{-2}$ and corresponds to the diagonal of the graph. Besides a small artifact close to the cut-off distance (only visible in the graph for $R_{C}=1.2$ and $1.6 \mathrm{~nm}$ ) and a slight offset (only visible in the graph for $R_{C}=1.2$ ), the curves are nearly indistinguishable from each other and from the Born polarization. In the main graph, the differences between $p(r)$ and $p_{\text {Born }}(r)$ are displayed as a function of $r^{-2}$ for the same values of $R_{C}$. In the short distance limit (right side of the graph), convergence towards the Born polarization is evident although slow. However, the differences are of rather small magnitude. For example, the relative difference at the surface of the ion, $\left[p_{\text {Born }}\left(R_{I}\right)-p\left(R_{I}\right)\right] / p_{\text {Born }}\left(R_{I}\right)$, evaluates to $0.91,0.29,0.12$, or $0.06 \%$ for $R_{C}=1.2,1.6,2.0$ or $2.4 \mathrm{~nm}$. In the long distance limit (left side of the graph), the approximate $r^{-2}$ evolution of $p(r)-p_{\text {Born }}(r)$ can also be observed. The maximal error in the polarization occurs exactly at the cut-off distance. Increasing the cut-off distance rapidly reduces the magnitude of this error, the difference $p_{\text {Born }}\left(R_{C}\right)-p\left(R_{C}\right)$ evaluating to $-0.021,-0.009,-0.004$, $-0.003 e \mathrm{~nm}^{-2}$ for $R_{C}=1.2,1.6,2.0$, or $2.4 \mathrm{~nm}$. These results indicate that for large-enough cut-off distances, the $\mathrm{RF} / \mathrm{NPBC}$ scheme provides an essentially correct representation of the polarization around a spherical ion, in both the short- and long-distance ranges.

\section{APPENDIX B: SELF-ENERGY TERM FOR CUT-OFF-BASED INTERACTION FUNCTIONS}

Here it is shown that when an effective cut-off-based interaction function is used to handle electrostatic interactions in an explicit-solvent simulation, a charge self-energy term should be included into the total electrostatic energy of the system to ensure a fast convergence of ionic solvation free energies towards the Born result in the limit of large cut-off distances. Generalizing this observation to the case of more complex molecular systems, a new definition [Eq. (B4)] is proposed for the electrostatic interaction energy in simulations employing the Barker-Watts reaction-field scheme.

Consider an effective cut-off-based electrostatic interaction function where the potential generated at $\mathbf{r}$ by a unit charge at the origin is given by

$$
\psi(r)=\frac{1}{4 \pi \epsilon_{o}} H(R-r)\left[r^{-1}+\widetilde{\psi}(r)\right],
$$

where $R$ is the cut-off radius, chosen smaller than half the smallest dimension of the computational box. It is further assumed that (i) the interaction function vanishes at $r=R$, i.e., $\psi(R)=0$, (ii) $\widetilde{\psi}$ is a sum of terms of the form $R^{-l-1} r^{l}$ with $l \geqslant 0$, and (iii) the polarization around a spherical ion converges to the Born polarization [Eq. (26)] in the limit of an infinite cut-off distance. For example, as shown in Appendix A, the Barker-Watts interaction function [Eq. (2)] satisfies the three conditions irrespective of the value of $\alpha$. In fact, there is some hint that the form of Eq. (B1) and the second condition automatically imply the third one. ${ }^{60}$ 
Under these assumptions and for large-enough cut-off distances, the polarization within the cut-off sphere of an ion should be quite close to the Born polarization [see Fig. 3(b) for the RF scheme]. Using the Born polarization as an approximation in this case, one may estimate the corresponding ionic solvation free energy. Combining Eq. (10) with $\mathbf{V}(\mathbf{r})$ $=-q_{I} \nabla \psi(r)$ and Eq. (26), one obtains the approximate expression

$$
\begin{aligned}
\Delta G_{\mathrm{solv}} & \approx \frac{q_{I}^{2}}{8 \pi} \frac{\epsilon_{s}-1}{\epsilon_{s}} \int_{R_{I}}^{R} d r 4 \pi \frac{\mathbf{r}}{r} \cdot \nabla \psi(r) \\
& =\frac{q_{I}^{2}}{2} \frac{\epsilon_{s}-1}{\epsilon_{s}}\left[\psi(R)-\psi\left(R_{I}\right)\right] \\
& =\Delta G_{\mathrm{Born}}-\frac{q_{I}^{2}}{8 \pi \epsilon_{o}} \frac{\epsilon_{s}-1}{\epsilon_{s}} \widetilde{\psi}\left(R_{I}\right),
\end{aligned}
$$

where we used $\psi(R)=0$ and inserted Eq. (28). In the RF case, estimates based on this equation can be compared to the data in Table II. For example, for $\epsilon_{s}=78, q_{I}=1 e, R_{I}$ $=0.2 \mathrm{~nm}$ and $R_{\mathrm{IS}}=R_{\mathrm{SS}}=1.2 \mathrm{~nm}$, Eq. (B2) [using Eq. (2) with $\alpha=1$ ] gives an estimate of $-258.0 \mathrm{~kJ} \mathrm{~mol}^{-1}$ (including $27.8 \mathrm{~kJ} \mathrm{~mol}^{-1}$ for the $\alpha$-dependent contribution), to be compared with the numerical value of $-259.2 \mathrm{~kJ} \mathrm{~mol}^{-1}$ (including $26.2 \mathrm{~kJ} \mathrm{~mol}^{-1}$ for the $\alpha$-dependent contribution) in Table II. If $\widetilde{\psi}$ is a sum of terms of the form $R^{-l-1} r^{l}$ with $l$ $\geqslant 0, \widetilde{\psi}\left(R_{I}\right)$ can be approximated by $\widetilde{\psi}(0)$ in the limit of large cut-off radii and small ions. In this case, Eq. (B2) shows that the ionic solvation free energy computed from an explicitsolvent simulation employing a cut-off-based interaction function will converge significantly faster towards the Born result upon increasing the cut-off distance if a self-energy term of the form

$$
\Delta G_{\text {self }}=\frac{q_{I}^{2}}{8 \pi \epsilon_{o}} \frac{\epsilon_{s}-1}{\epsilon_{s}} \widetilde{\psi}(0)
$$

is included in the electrostatic energy of the system. Note that $\Delta G_{\text {self }}$ converges towards zero in the limit $R \rightarrow \infty$. However, because this term is generally large and converges only as $R^{-1}$, its inclusion makes a significant difference even for relatively large cut-off radii.

Generalizing this observation to more complex molecular systems suggests that a charge self-energy term should be included in explicit-solvent molecular dynamics simulations employing any effective cut-off-based electrostatic interaction function. Intuitively, this term may be interpreted as the reversible work required to individually charge the atoms when they are at infinite separation. This work excludes the (infinite) Coulombic self-energy, but retains the contribution arising from the non-Coulombic term associated with $\widetilde{\psi}$ in Eq. (B1).

In the specific case of the Barker-Watts reaction-field method [Eq. (2)], a reasonable expression for the total electrostatic energy $U_{\mathrm{BW}}$ could be

$$
\begin{aligned}
U_{\mathrm{BW}}= & \sum_{i} \sum_{j>i, j \notin \operatorname{excl}(i)} q_{i} q_{j} \psi_{\mathrm{BW}}\left(\bar{r}_{i j}\right) \\
& +\frac{1}{4 \pi \epsilon_{o}}\left\{\sum_{i} \sum_{j>i, j \in \operatorname{excl}(i)} q_{i} q_{j} \widetilde{\psi}_{\mathrm{BW}}\left(\bar{r}_{i j}\right)\right. \\
& \left.+\frac{1}{2} \widetilde{\psi}_{\mathrm{BW}}(0)\left[\sum_{i} q_{i}^{2}-\epsilon_{s}^{-1}\left(\sum_{i} q_{i}\right)^{2}\right]\right\},
\end{aligned}
$$

where $\overline{\mathbf{r}}_{i j}$ is the minimum-image vector corresponding to $\mathbf{r}_{i j}$, $\operatorname{excl}(i)$ denotes the exclusion list of atom $i$ (the distance between any two excluded atoms is assumed to be smaller than $R$ ), and

$$
\widetilde{\psi}_{\mathrm{BW}}(r)=\frac{\alpha r^{2}}{2 R^{3}}-\frac{\alpha+2}{2 R},
$$

with $\alpha$ defined by Eq. (3). Note that current simulation programs (e.g., GROMOS ${ }^{92}$ and GROMACS ${ }^{93}$ ) typically restrict the implementation of the Barker-Watts reaction-field method to the first term in Eq. (B4). The second term is explicitly included here because so-called excluded neighbors (usually first and second covalent neighbors) should only be excluded from the summation of the Coulombic $\left(r^{-1}\right)$ contribution, but not of the reaction-field ( $\left.\widetilde{\psi}_{\mathrm{BW}}\right)$ contribution. The form of the third term has been chosen for consistency in the context of small molecules (compared to the cut-off radius and unitcell size). For a small molecule (or ion) gathered by periodicity around its center, $\bar{r}_{i j}$ can be replaced by $r_{i j}$ in Eq. (B4) and the Heaviside function involved in $\psi_{\mathrm{BW}}$ can be omitted. In this case, the reaction-field (non-Coulombic) contribution contribution to $U_{\mathrm{BW}}$ can be written

$U_{\mathrm{BW}}^{\mathrm{rf}}=\frac{1}{8 \pi \epsilon_{o}}\left\{\frac{\alpha}{R^{3}} \sum_{i} \sum_{j>i} q_{i} q_{j} r_{i j}^{2}+\frac{\epsilon_{s}-1}{\epsilon_{s}} \widetilde{\psi}_{\mathrm{BW}}(0)\left(\sum_{i} q_{i}\right)^{2}\right\}$.

For a neutral molecule, one has

$$
U_{\mathrm{BW}}^{\mathrm{rf}, \mathrm{dip}}=-\frac{1}{8 \pi \epsilon_{o}} \frac{\alpha \mu^{2}}{R^{3}},
$$

where $\mu$ is the molecular dipole moment, which matches the Onsager expression for a dipole in a spherical cavity ${ }^{94}$ provided that adjusted boundary conditions $\left[\epsilon^{\prime}=\epsilon_{s}\right.$ in Eq. (3) $]$ are applied. For a monoatomic ion, one has $U_{\mathrm{BW}}^{\mathrm{rf} \text {,ion }}=\Delta G_{\text {self }}$, i.e., the self-energy term suggested by Eq. (B3). Note that the last term in Eq. (B4) only affects the energy of the system, but does not induce atomic forces. However, it may be essential to include it in free-energy calculations involving alterations of the atomic partial charges.

In the specific case of a single ion, the inclusion of such a self-energy term should substantially reduce the error on ionic solvation free energies computed from explicit-solvent simulations with finite cut-off distances. This can be seen from the corresponding corrected values $\Delta G_{\text {solv }}^{\text {corr }}=\Delta G_{\text {solv }}$ $+\Delta G_{\text {self }}$ reported in Table II. In the RF case, taking the same example as above $\left(\epsilon_{s}=78, q_{I}=1 e, R_{I}=0.2 \mathrm{~nm}\right.$ and $R_{\mathrm{IS}}$ $\left.=R_{\mathrm{SS}}=1.2 \mathrm{~nm}\right), \Delta G_{\text {solv }}^{\text {Born }}$ evaluates $-342.9 \mathrm{~kJ} \mathrm{~mol}^{-1}$, to be compared with an estimate $\Delta G_{\text {solv }}^{\text {corr }}$ of $-344.9 \mathrm{~kJ} \mathrm{~mol}^{-1}$. The corresponding estimate for the ST case, $-363.4 \mathrm{~kJ} \mathrm{~mol}^{-1}$, is significantly less accurate. This is probably due to the poorer 
agreement between the polarization within the cut-off sphere of an ion and the Born polarization in this case [see Fig. $3(\mathrm{a})]$.

${ }^{1}$ M. Karplus and J. A. McCammon, Nat. Struct. Biol. 9, 646 (2002).

${ }^{2}$ X. Daura, A. Glättli, P. Gee, C. Peter, and W. F. van Gunsteren, Adv. Protein Chem. 62, 341 (2002)

${ }^{3}$ T. E. Cheatham, III and P. A. Kollman, Annu. Rev. Phys. Chem. 51, 435 (2000).

${ }^{4}$ L. Saiz, S. Bandyopadhyay, and M. L. Klein, Biosci Rep. 22, 151 (2002).

${ }^{5}$ S. B. Engelsen, C. Monteiro, C. Hervé de Penhoat, and S. Pérez, Biophys. Chem. 93, 103 (2001).

${ }^{6}$ W. F. van Gunsteren and H. J. C. Berendsen, Angew. Chem., Int. Ed. Engl. 29, 992 (1990).

${ }^{7}$ M. P. Allen and D. J. Tildesley, Computer Simulation of Liquids (Oxford University Press, New York, 1987).

${ }^{8}$ B. R. Brooks, R. E. Bruccoleri, B. D. Olafson, D. J. States, S. Swaminathan, and M. Karplus, J. Comput. Chem. 4, 187 (1983).

${ }^{9}$ C. L. Brooks III, B. M. Pettitt, and M. Karplus, J. Chem. Phys. 83, 5897 (1985).

${ }^{10}$ K. S. Kim, Chem. Phys. Lett. 156, 261 (1989).

${ }^{11}$ R. J. Loncharich and B. R. Brooks, Proteins: Struct., Funct., Genet. 6, 32 (1989).

${ }^{12}$ P. J. Steinbach and B. R. Brooks, J. Comput. Chem. 15, 667 (1994).

${ }^{13}$ J. Norberg and L. Nilsson, Biophys. J. 79, 1537 (2000).

${ }^{14}$ J. A. Barker and R. O. Watts, Mol. Phys. 26, 789 (1973).

${ }^{15}$ I. G. Tironi, R. Sperb, P. E. Smith, and W. F. van Gunsteren, J. Chem. Phys. 102, 5451 (1995).

${ }^{16}$ P. H. Hünenberger and W. F. van Gunsteren, J. Chem. Phys. 108, 6117 (1998).

${ }^{17}$ G. Hummer and D. M. Soumpasis, Phys. Rev. E 49, 591 (1994).

${ }^{18}$ G. Hummer and D. M. Soumpasis, J. Phys.: Condens. Matter 6, A141 (1994).

${ }^{19}$ G. Hummer, L. R. Pratt, and A. E. Garcia, J. Phys. Chem. 100, 1206 (1996).

${ }^{20}$ P. P. Ewald, Ann. Phys. (Leipzig) 64, 253 (1921).

${ }^{21}$ R. W. Hockney and J. W. Eastwood, Computer Simulation Using Particles (Institute of Physics, Bristol, 1981).

${ }^{22}$ T. Darden, D. York, and L. Pedersen, J. Chem. Phys. 98, 10089 (1993).

${ }^{23}$ U. Essmann, L. Perera, M. L. Berkowitz, T. Darden, H. Lee, and L. G. Pedersen, J. Chem. Phys. 103, 8577 (1995).

${ }^{24}$ M. Neumann, Mol. Phys. 50, 841 (1983).

${ }^{25}$ M. Neumann, O. Steinhauser, and G. S. Pawley, Mol. Phys. 52, 97 (1984).

${ }^{26}$ R. H. Wood, J. Chem. Phys. 103, 6177 (1995).

${ }^{27}$ N. A. Baker, P. H. Hünenberger, and J. A. McCammon, J. Chem. Phys. 110, 10679 (1999).

${ }^{28}$ N. A. Baker, P. H. Hünenberger, and J. A. McCammon, J. Chem. Phys. 113, 2510 (1999).

${ }^{29}$ C. L. Brooks III, J. Phys. Chem. 90, 6680 (1986).

${ }^{30}$ C. L. Brooks III, J. Chem. Phys. 86, 5156 (1987).

${ }^{31}$ J. D. Madura and B. M. Pettitt, Chem. Phys. Lett. 150, 105 (1988).

${ }^{32}$ T. P. Straatsma and H. J. C. Berendsen, J. Chem. Phys. 89, 5876 (1988).

${ }^{33}$ S. G. Kalko, G. Sese, and J. A. Padro, J. Chem. Phys. 104, 9578 (1996).

${ }^{34}$ H. Resat and J. A. McCammon, J. Chem. Phys. 104, 7645 (1996).

${ }^{35}$ H. Resat and J. A. McCammon, J. Chem. Phys. 108, 9617 (1998).

${ }^{36}$ B. M. Pettitt and P. J. Rossky, J. Chem. Phys. 84, 5836 (1986).

${ }^{37}$ L. X. Dang and B. M. Pettitt, J. Phys. Chem. 94, 4303 (1990).

${ }^{38}$ E. Guàrdia, R. Rey, and J. A. Padró, J. Chem. Phys. 95, 2823 (1991).

${ }^{39}$ L. X. Dang, B. M. Pettitt, and P. J. Rossky, J. Chem. Phys. 96, 4046 (1992).

${ }^{40}$ G. Hummer, D. M. Soumpasis, and M. Neumann, Mol. Phys. 81, 1155 (1994).

${ }^{41}$ J. S. Bader and D. Chandler, J. Phys. Chem. 96, 6423 (1992).

${ }^{42}$ R. A. Friedman and M. Mezei, J. Chem. Phys. 102, 419 (1994).

${ }^{43}$ G. S. Del Buono, F. E. Figueirido, and R. M. Levy, Chem. Phys. Lett. 263, 521 (1996).

${ }^{44}$ X. Rozanska and C. Chipot, J. Chem. Phys. 112, 9691 (2000).

${ }^{45}$ H. Schreiber and O. Steinhauser, Biochemistry 31, 5856 (1992).

${ }^{46}$ H. Schreiber and O. Steinhauser, Chem. Phys. 168, 75 (1992).

${ }^{47}$ H. Schreiber and O. Steinhauser, J. Mol. Biol. 228, 909 (1992).

${ }^{48}$ P. E. Smith and B. M. Pettitt, J. Chem. Phys. 95, 8430 (1991).

${ }^{49}$ G. S. Del Buono, T. S. Cohen, and P. J. Rossky, J. Mol. Liq. 60, 221 (1994).
${ }^{50}$ L. Perera, U. Essmann, and M. L. Berkowitz, J. Chem. Phys. 102, 450 (1994).

${ }^{51}$ S. Boresch and O. Steinhauser, J. Chem. Phys. 115, 10780 (2001).

${ }^{52}$ P. H. Hünenberger, in Simulation and Theory of Electrostatic Interactions in Solution: Computational Chemistry, Biophysics, and Aqueous Solution, edited by G. Hummer and L. R. Pratt (American Institute of Physics, New York, 1999), pp. 17-83.

${ }^{53}$ M. Deserno and C. Holm, J. Chem. Phys. 109, 7678 (1998).

${ }^{54}$ M. Deserno and C. Holm, J. Chem. Phys. 109, 7694 (1998).

${ }^{55}$ G. Hummer, L. R. Pratt, and A. E. Garcia, J. Phys. Chem. A 102, 7885 (1998).

${ }^{56}$ M. Kastenholz and P. H. Hünenberger, J. Phys. Chem B (to be published).

${ }^{57}$ P. H. Hünenberger and J. A. McCammon, J. Chem. Phys. 110, 1856 (1999).

${ }^{58}$ P. H. Hünenberger and J. A. McCammon, Biophys. Chem. 78, 69 (1999).

${ }^{59}$ W. Weber, P. H. Hünenberger, and J. A. McCammon, J. Phys. Chem. B 104, 3668 (2000).

${ }^{60}$ C. Peter, W. F. van Gunsteren, and P. H. Hünenberger, J. Chem. Phys. (to be published).

${ }^{61}$ S. Boresch and O. Steinhauser, J. Chem. Phys. 111, 8271 (1999).

${ }^{62}$ S. Boresch and O. Steinhauser, J. Chem. Phys. 115, 10793 (2001).

${ }^{63}$ M. Marchi, D. Borgis, N. Levy, and P. Ballone, J. Chem. Phys. 114, 4377 (2001).

${ }^{64}$ C. Peter, W. F. van Gunsteren, and P. H. Hünenberger, J. Chem. Phys. 116, 7434 (2002)

${ }^{65}$ J. D. Madura, M. E. Davis, M. K. Gilson, R. C. Wade, B. A. Luty, and J. A. McCammon, in Reviews in Computational Chemistry, Vol. 4, edited by K. B. Lipkowitz and D. B. Boyd (VCH, New York, 1994), pp. 229-267.

${ }^{66}$ B. Honig, K. Sharp, and A.-S. Yang, J. Phys. Chem. 97, 1101 (1993).

${ }^{67}$ M. K. Gilson, Curr. Opin. Struct. Biol. 5, 216 (1995).

${ }^{68}$ B. Honig and A. Nicholls, Science 268, 1144 (1995).

${ }^{69}$ F. Figueirido, G. S. Del Buono, and R. M. Levy, J. Phys. Chem. B 101, 5622 (1997).

${ }^{70}$ G. Hummer, L. R. Pratt, and A. E. Garcia, J. Chem. Phys. 107, 9275 (1997).

${ }^{71}$ S. Sakane, H. S. Ashbaugh, and R. H. Wood, J. Phys. Chem. B 102, 5673 (1998).

${ }^{72}$ G. Hummer, L. R. Pratt, A. E. Garcia, B. J. Berne, and S. W. Rick, J. Phys. Chem. B 101, 3017 (1997).

${ }^{73}$ H. S. Ashbaugh and R. H. Wood, J. Chem. Phys. 106, 8135 (1997).

${ }^{74}$ J. Åvist and T. Hansson, J. Phys. Chem. B 102, 3837 (1998).

${ }^{75}$ H. S. Ashbaugh and R. H. Wood, J. Phys. Chem. B 102, 3844 (1998).

${ }^{76}$ G. Hummer, L. R. Pratt, A. E. Garcia, S. Garde, B. J. Berne, and S. W. Rick, J. Phys. Chem. B 102, 3841 (1998).

${ }^{77}$ Y. N. Vorobjev and J. Hermans, J. Phys. Chem. 103, 10234 (1999).

${ }^{78}$ T. Darden, D. Pearlman, and L. G. Pedersen, J. Chem. Phys. 109, 10921 (1998).

${ }^{79}$ Y. Y. Sham and A. Warshel, J. Chem. Phys. 109, 7940 (1998).

${ }^{80}$ H. Resat, M. Mezei, and J. A. McCammon, J. Phys. Chem. 100, 1426 (1996).

${ }^{81}$ F. Figueirido, G. S. Del Buono, and R. M. Levy, J. Chem. Phys. 103, 6133 (1995).

${ }^{82}$ L. R. Pratt, G. Hummer, and A. E. Garciá, Biophys. Chem. 51, 147 (1994).

${ }^{83}$ A. Kovalenko and F. Hirata, J. Chem. Phys. 112, 10403 (2000).

${ }^{84}$ A. Masunov and T. Lazaridis, J. Am. Chem. Soc. 125, 1722 (2003).

${ }^{85}$ M. Neumann and O. Steinhauser, Mol. Phys. 57, 97 (1986).

${ }^{86}$ M. Born, Z. Phys. 1, 45 (1920).

${ }^{87}$ J.-C. Soetens, C. Millot, C. Chipot, G. Jansen, J. G. Ángyán, and B. Maigret, J. Phys. Chem. B 101, 10910 (1997).

${ }^{88}$ L. Degrève and F. L. B. da Silva, J. Chem. Phys. 110, 3070 (1999).

${ }^{89}$ D. Sardella Vieira and L. Degrève, J. Mol. Struct. 580, 127 (2002).

${ }^{90}$ G. Hummer, D. M. Soumpasis, and M. Neumann, Mol. Phys. 77, 769 (1992).

${ }^{91}$ C. Satheesan Babu, P.-K. Yang, and C. Lim, J. Biol. Phys. 28, 95 (2002).

${ }^{92}$ W. F. van Gunsteren, S. R. Billeter, A. A. Eising, P. H. Hünenberger, P. Krüger, A. E. Mark, W. R. P. Scott, and I. G. Tironi, Biomolecular Simulation: The GROMOS96 Manual and User Guide (Verlag der Fachvereine, Zürich, 1996).

${ }^{93}$ H. J. C. Berendsen, D. van der Spoel, and R. van Drunen, Comput. Phys. Commun. 91, 43 (1995).

${ }^{94}$ L. Onsager, J. Am. Chem. Soc. 58, 1486 (1936).

${ }^{95}$ J. Warwicker and H. C. Watson, J. Mol. Biol. 157, 671 (1982).

${ }^{96}$ P. Auffinger and D. L. Beveridge, Chem. Phys. Lett. 234, 413 (1995). 